Y. Ito and I. Kubo

Nagoya Math. J.

Vol. 111 (1988), 41-84

\title{
CALCULUS ON GAUSSIAN AND POISSON WHITE NOISES
}

\author{
YOSHIFUSA ITO AND IZUMI KUBO
}

\section{§1. Introduction}

Recently one of the authors has introduced the concept of generalized Poisson functionals and discussed the differentiation, renormalization, stochastic integrals etc. ([8], [9]), analogously to the works of T. Hida ([3], [4], [5]). Here we introduce a transformation $\mathscr{S}_{P}$ for Poisson fnnctionals with the idea as in the case of Gaussian white noise (cf. [10], [11], [12], [13]). Then we can discuss the differentiation, renormalization, multiple Wiener integrals etc. in' a way completely parallel with the Gaussian case. The only one exceptional point, which is most significant, is that the multiplications are described by

$$
\begin{array}{ll}
x^{G}(t) \cdot=\partial_{t}^{*}+\partial_{t} & \text { for the Gaussian case, } \\
x^{P}(t) \cdot=\left(\partial_{t}^{*}+1\right)\left(\partial_{t}+1\right) & \text { for the Poisson case, }
\end{array}
$$

as will be stated in Section 5. Conversely, those formulae characterize the types of white noises.

In Section 2, we will define Gaussian and Poisson white noises on a general parameter space $T$, which is a separable topological space with a $\sigma$-finite non-atomic Borel measure $\nu$. Let $\mathscr{E} \subset L^{2}(T, \nu) \subset \mathscr{E} *$ be a Gel'fand triplet satisfying the assumptions [A.1], [A.2], [A.3] in Section 2. Then the measure of Gaussian white noise $\mu_{G}$ and the measure of Poisson white noise $\mu_{P}$ are characterized respectively by their Fourier transforms

$$
\begin{aligned}
& \int_{\varepsilon^{*}} \exp [i\langle x, \xi\rangle] d \mu_{G}(x)=\exp \left[-\frac{1}{2} \int_{T}|\xi(t)|^{2} d \nu(t)\right], \\
& \left.\int_{\varepsilon^{*}} \exp [i\langle x, \xi\rangle] d \mu_{P}(x)=\exp \left[\int_{T}\left(\exp \left[i \xi^{\prime}(t)\right]-1\right) d \nu^{\prime} t\right)\right],
\end{aligned}
$$

with $\xi$ in $\mathscr{E}$. Then we introduce transformations $\mathscr{S}_{G}$ from $L^{2}\left(\mathscr{E} *, \mu_{G}\right)$ and $\mathscr{S}_{P}$ from $L^{2}\left(\mathscr{E}^{*}, \mu_{P}\right)$ to the same space $\mathscr{F}^{(0)}$ which is a Hilbert space with the reproducing kernel $\exp [\langle\xi, \eta\rangle], \eta \in \mathscr{E}$.

Received September 1, 1986. 
In Section 3, we will see fundamental properties of the space $\mathscr{F}^{(0)}$. Then we will construct a new Gel'fand triplet $\mathscr{F} \subset \mathscr{F}^{(0)} \subset \mathscr{F}^{*}$ in connection with the structure of the basic Gel'fand triplet $\mathscr{E} \subset L^{2}(T, \nu) \subset \mathscr{E} *$. Propositions, which will be used in later sections, are prepared. Any functional $U(\xi)$ in $\mathscr{F}^{*}$ is Fréchet differentiable (Theorem 3.9). Moreover, if $U(\xi)$ is in $\mathscr{F}$, then the $n$-th Fréchet derivative $U^{(n)}\left(\xi ; \eta_{1}, \eta_{2}, \cdots, \eta_{n}\right)$ defines an element $U^{(n)}(\xi ; \cdot)$ of $\mathscr{E}^{\hat{\otimes} n}$ such that

$$
U^{(n)}\left(\xi ; \eta_{1}, \eta_{2}, \cdots, \eta_{n}\right)=\int_{T^{n}} d \nu^{n}(t) U^{(n)}(\xi ; t) \eta_{1}\left(t_{1}\right) \eta_{2}\left(t_{2}\right) \cdots \eta_{n}\left(t_{n}\right)
$$

and that $U^{(n)}(\xi ; t)$ belongs to $\mathscr{F}$ for fixed $t=\left(t_{1}, t_{2}, \cdots, t_{n}\right) \in T^{n}$ (Theorem 3.9, Corollary 3.14). The mapping $U(\tilde{\xi}) \rightarrow\left\{\frac{1}{n !} U^{(n)}(0 ; \cdot)\right\}$ defines an isomorphism from the Gel'fand triplet $\mathscr{F} \subset \mathscr{F}^{(0)} \subset \mathscr{F}^{*}$ to that of Fock's spaces $\exp [\hat{\otimes} \mathscr{E}] \subset \exp \left[\hat{\otimes} L^{2}(T, \nu)\right] \subset \exp [\hat{\otimes} \mathscr{E} *]$.

In Section 4, a Gel'fand triplet $\mathscr{H}_{X} \subset \mathscr{H}_{X}^{(0)} \subset \mathscr{H}_{X}^{*}$ will be induced from $\mathscr{F} \subset \mathscr{F}^{(0)} \subset \mathscr{F}^{*}$ through the mapping $\mathscr{S}_{X}, X=G, P$, respectively. A continuous operator $\partial_{t}$ on $\mathscr{H}_{X}$ is defined by

$$
\partial_{t} \varphi \equiv \mathscr{S}_{X}^{-1}\left(\frac{\delta}{\delta \xi(t)}\left(\mathscr{S}_{X} \varphi\right)(\xi)\right)
$$

Then its dual $\partial_{t}^{*}$ is a continuous operator on $\mathscr{H}_{x}^{*}$, which satisfies

$$
\partial_{t}^{*} \varphi=\mathscr{S}_{X}^{-1}\left(\xi(t) \cdot\left(\mathscr{S}_{X} \varphi\right)(\xi)\right) \text {. }
$$

For $f_{n} \in L^{2}\left(T^{n}, \nu^{n}\right)$, denote by $I^{G}\left(f_{n}\right)$ and by $I^{P}\left(f_{n}\right)$ the multiple Wiener integrals with respect to the Gaussian white noise and the Poisson white noise, respectively. Then $I^{X}\left(f_{n}\right)$ has the expression

$$
I^{X}\left(f_{n}\right)=\int_{T^{n}} d \nu^{n}(t) f_{n}(t) \partial_{t_{1}}^{*} \partial_{t_{2}}^{*} \cdots \partial_{t_{n}}^{*} 1
$$

(see Theorem 4.7). Some estimations of norms of operators related to $\partial_{t}$ and $\partial_{t}^{*}$ will be given.

In Section 5, we shall characterize the respective white noises by means of multiplication operators, as stated above (see Theorem 5.3, Theorem 5.4, Theorem 5.5).

In Section 6, we note that we can use the concept of Wick's normal ordering : :, since $\left\{\partial_{t}, \partial_{t}^{*} ; t \in T\right\}$ satisfy the canonical commutation relations. By using it, we have a new expression of the multiple Wiener integral $I_{n}^{X}\left(f_{n}\right)$ as 


$$
I_{n}^{X}\left(f_{n}\right)=\int_{T^{n}} d \nu^{n}(\boldsymbol{t}) f_{n}(\boldsymbol{t}):\left(x\left(t_{1}\right) \cdot-\left\langle x\left(t_{1}\right)\right\rangle\right) \cdots\left(x\left(t_{n}\right) \cdot-\left\langle x\left(t_{n}\right)\right\rangle\right): 1
$$

in $\mathscr{H}_{X}^{*}, X=G, P$. The expression is convenient, because the relation

$$
I_{n}^{X}\left(f_{n}\right) \varphi=\int_{T^{n}} d \nu^{n}(\boldsymbol{t}) f_{n}(\boldsymbol{t}):\left(x\left(t_{1}\right) \cdot-\left\langle x\left(t_{1}\right)\right\rangle\right) \cdots\left(x\left(t_{n}\right) \cdot-\left\langle x\left(t_{n}\right)\right\rangle\right): \varphi
$$

holds in $\mathscr{H}_{X}^{*}$ (Theorem 6.4). The renormalization in the case of Gaussian by $\mathrm{T}$. Hida (or in the case of Poisson by one of the authors) coincides with Wick's normal ordering after operating on the constant 1, which may be called the vacuum (cf. [3], [9], [11]).

In Section 7, we will remark that the operator $\partial_{t}$ corresponds to the difference $\Delta_{t} \varphi \equiv \varphi\left(x+\delta_{t}\right)-\varphi(x)$ and the transformation $\mathscr{S}_{P}$ is closely related to the semi-group with the generator $\Delta_{t}$ in the Poisson case. In the Gaussian case $\partial_{t}$ corresponds to Gâteaux derivative in the direction of $\delta_{t}$ (see [13]).

\section{§2. Gaussian and Poisson white noises}

First of all, we give a setting of calculus on a Gaussian white noise (cf. [10], [11], [12]). Let $T$ be a separable topological space with a $\sigma$-finite non-atomic Borel measure $\nu$. Let $\mathscr{E}$ be a dense linear subset of $L^{2}(T, \nu)$ and let $\left\{(\xi, \eta)_{p} ; \xi, \eta \in \mathscr{E}\right\}_{p \geq 0}$ be a consistent sequence of inner products such that

$$
\rho\|\xi\|_{p+1} \geq\|\xi\|_{p} \quad \text { for any } \xi \in \mathscr{E}, p \geq 0,
$$

with a fixed $\rho \in(0,1)$ and

$$
(\xi, \eta)_{0} \equiv \int_{T} \xi(t) \eta(t) d \nu(t)
$$

Let us identify $E_{0}=L^{2}(T, \nu)$ with its dual. Let $E_{p}$ be the completion of $\mathscr{E}$ with respect to the inner product $(,)_{p}$ and let $E_{-p}$ be the dual of $E_{p}$ with inner product $(,)_{-p}$, for $p \geq 1$. Then we have inclusions $E_{p+1} \subset E_{p}$, $p \in Z$. Suppose that $\mathscr{E}=\bigcap_{p} E_{p}$ and topologize $\mathscr{E}$ as the projective limit of $E_{p}$ as $p \rightarrow \infty$. Let $\mathscr{E}^{*}$ be the dual of $\mathscr{E}$, then $\mathscr{E} *$ is the inductive limit of $E_{p}$ as $p \rightarrow-\infty$. We denote by $\langle$,$\rangle the canonical bilinear forms between$ any dual pairs. Then obviously, $\langle\xi, \eta\rangle=(\xi, \eta)_{0}$ holds if $\xi, \eta \in E_{0}$. Additionally, we assume the following:

[A.1] The injection $E_{1} \subseteq E_{0}$ is traceable; that is, the evaluation map $\delta_{t}: \xi \rightarrow \xi(t)$ gives a continuous mapping $t \rightarrow \delta_{t}$ from $T$ into $E_{-1}$. Moreover, 


$$
\|\delta\|^{2} \equiv \int_{T}\left\|\delta_{t}\right\|_{-1}^{2} d \nu(t)<\infty
$$

Then the injection $E_{1} \subseteq E_{0}$ is a Hilbert-Schmidt operator (see [10]). Hence, by Bochner-Minlos-Sazanov's theorem, we can find a probability measure $\mu_{G}$ on $\mathscr{E}^{*}$ such that

$$
\int_{\sigma^{*}} \exp [i\langle x, \xi\rangle] d \mu_{G}(x)=\exp \left[-\frac{1}{2}\|\xi\|_{0}^{2}\right] \text {. }
$$

Let us denote $L^{2}\left(\mathscr{E}^{*}, \mu_{G}\right)$ by $\left(L_{G}^{2}\right)$. Then the following transformation $\mathscr{S}_{G}$ gives an isomorphism from $\left(L_{G}^{2}\right)$ onto $\mathscr{F}^{(0)}$ which is the Hilbert space with reproducing kernel $\exp [\langle\xi, \eta\rangle], \xi, \eta \in \mathscr{E}:$ For $\varphi(x) \in\left(L_{G}^{2}\right)$, define

$$
\begin{aligned}
\left(\mathscr{S}_{G} \varphi\right)(\xi) & =\int_{\sigma^{*}} \varphi(x+\xi) d \mu_{G}(x) \\
& =\int_{\sigma^{*}} \varphi(x) \exp \left[\langle x, \xi\rangle-\frac{1}{2}\|\xi\|_{0}^{2}\right] d \mu_{G}(x),
\end{aligned}
$$

by using a theorem on the density of Gaussian measure (see [14]). Actually, put

$$
f^{G}(\xi)=f^{G}(\xi ; x) \equiv \exp \left[\langle x, \xi\rangle-\frac{1}{2}\|\xi\|_{0}^{2}\right] \quad \text { for } \xi \in \mathscr{E} .
$$

Then we have

$$
\begin{aligned}
& \left(\mathscr{S}_{G} f^{G}(\eta)\right)(\xi)=\exp [\langle\xi, \eta\rangle], \\
& \left(\mathscr{S}_{G} \varphi\right)(\xi)=\left(\varphi(x), f^{G}(\xi ; x)\right)_{\left(L_{G}^{2}\right)}, \\
& \left(f^{G}(\xi ; x), f^{G}(\eta ; x)\right)_{\left(L_{G}^{2}\right)}=\exp [\langle\xi, \eta\rangle] .
\end{aligned}
$$

Since $\left\{f^{G}(\eta ; x) ; \eta \in \mathscr{E}\right\}$ is a total subset of $\left(L_{G}^{2}\right)$; that is, the set of all linear combinations is dense in $\left(L_{G}^{2}\right)$, the above formulae imply that $\exp [\langle\xi, \eta\rangle]$ is the reproducing kernel.

Let us introduce the Hermite's polynomials with parameter $\gamma$ by the generating function $\exp \left[\omega z-\frac{\gamma}{2} \omega^{2}\right]$;

$$
\sum_{n=0}^{\infty} \frac{1}{n !} \omega^{n} H_{n}(z ; \gamma) \equiv \exp \left[\omega z-\frac{\gamma}{2} \omega^{2}\right] \text {. }
$$

Then we have

$$
\mathscr{S}_{G}: H_{n}\left(\langle x, \eta\rangle ;\|\eta\|_{0}^{2}\right) \longrightarrow\langle\xi, \eta\rangle^{n}
$$


more generally,

$$
\mathscr{S}_{G}: H_{n}(\langle x, \eta\rangle ; \gamma) \longrightarrow H_{n}\left(\langle\xi, \eta\rangle ; \gamma-\|\eta\|_{0}^{2}\right) .
$$

Therefore we have

$$
\begin{aligned}
\left(\langle\xi, \eta\rangle^{n},\langle\xi, \zeta\rangle^{m}\right)_{\mathscr{F}(0)} & =\left(H_{n}\left(\langle x, \eta\rangle ;\|\eta\|_{0}^{2}\right), H_{m}\left(\langle x, \zeta\rangle ;\|\zeta\|_{0}^{2}\right)_{\left(L_{G}^{2}\right)}\right. \\
& =\delta_{n, m} n !\langle\xi, \eta\rangle^{n} .
\end{aligned}
$$

Now we come to Poisson white noise on $T$ with intensity $\nu$. To establish the calculus on Poisson white noise, it is convenient to require that the basic function space has an algebraic structure. Hence we assume additional assumptions:

[A.2] For $\xi, \eta \in \mathscr{E}, \xi \eta$ belongs to $\mathscr{E}$ and satisfies

$$
\|\xi \eta\|_{p} \leq C_{p} \cdot\|\xi\|_{p} \cdot\|\eta\|_{p} \quad \text { for } p \geq 1 \text {. }
$$

[A.3] The triplet $\mathscr{E} \subset L^{2}(T, \nu) \subset \mathscr{E}^{*}$ is a Gel'fand triplet and the evaluating map $\delta$ satisfies

$$
\|\delta\|_{\infty} \equiv \int_{T}\left\|\delta_{t}\right\|_{-1} d \nu(t)+\sup _{t \in T}\left\|\delta_{t}\right\|_{-1}<\infty .
$$

It must be noted that Schwartz space $\mathscr{S}\left(\boldsymbol{R}^{d}\right)$ admits a consistent sequence of inner products which satisfy the assumptions [A.1, 2, 3].

Since estimations

$$
\begin{aligned}
& |\xi(t)|=\left|\left\langle\delta_{t}, \xi\right\rangle\right| \leq\left\|\delta_{t}\right\|_{-1}\|\xi\|_{1}, \\
& |\xi(t)-\xi(s)| \leq\left\|\delta_{t}-\delta_{s}\right\|_{-1}\|\xi\|_{1},
\end{aligned}
$$

hold for $\xi \in E_{1}, \xi$ is a continuous bounded function belonging to $L^{1}(T, \nu) \cap L^{2}(T, \nu)$ with

$$
\|\xi\|_{L^{1}}+\sup _{t \in T}|\xi(t)| \leq\|\delta\|_{\infty}\|\xi\|_{-1} \text {. }
$$

Furthermore, $C_{P}(\xi) \equiv \exp \left[\int_{T}(\exp [i \xi(t)]-1) d \nu(t)\right]$ is a positive definite continuous functional on $E_{1}$. Hence there exists a probability measure $\mu_{P}$ on $\mathscr{E}^{*}$ such that

$$
\int_{\Theta^{*}} \exp [i\langle x, \xi\rangle] d \mu_{P}(x)=\exp \left[\int_{T}(\exp [i \xi(t)]-1) d \nu(t)\right]
$$

for any $\xi \in \mathscr{E}$. We call $\mu_{P}$ the measure of Poisson white noise with intensity $\nu$. Denote by $\left(L_{P}^{2}\right)$ the $L^{2}$-space $L^{2}\left(\mathscr{E}^{*}, \mu_{P}\right)$. To analyze $\left(L_{P}^{2}\right)$, we define 
an isomorphism $\mathscr{S}_{P}$ from $\left(L_{P}^{2}\right)$ onto the reproducing kernel Hilbert space $\mathscr{F}(0)$ introduced above by

$$
\left(\mathscr{S}_{P} \varphi\right)(\xi) \equiv \int_{\ell^{*}} \varphi(x) \exp \left[\langle x, \log (1+\xi)\rangle-\int_{T} \xi(u) d \nu(u)\right] d \mu_{P}(x)
$$

for $\xi \in \mathscr{E}$. Since $\log (1+\xi(t))$ does not belong to $\mathscr{E}$ in general, we therefore use a trick as follows. For simplicity of notation, we write

$$
\bar{\eta}=\int_{T} \eta(t) d \nu(t)
$$

Since $I^{P}(\eta)=I^{P}(\eta ; x) \equiv\langle x, \eta\rangle-\bar{\eta}, \eta \in \mathscr{E}$, is a random variable with mean zero and variance $\|\eta\|_{0}^{2}$, the mapping $I^{P}: \mathscr{E} \rightarrow\left(L_{P}^{2}\right)$ can be extended to an isometry from $E_{0}=L^{2}(T, \nu)$ into $\left(L_{P}^{2}\right)$. Then

$$
\int_{\sigma^{*}} \exp \left[i I^{P}(\eta ; x)\right] d \mu_{P}(x)=\exp \left[\int_{T}(\exp [i \eta(u)]-i \eta(u)-1) d \nu(u)\right]
$$

holds for $\eta \in E_{0}$. For a given Borel set $A$ with $\nu(A)<\infty$, we define a Poisson random measure $P(A)$ by

$$
\bar{P}(A) \equiv I^{P}\left(\chi_{A} ; x\right)^{\circ} \text { and } P(A) \equiv \bar{P}(A)+\nu(A) .
$$

Appealing to (2.13), we can see that $P(A)$ is a Poisson random variable with mean $\nu(A)$, i.e.

$$
\mu_{P}(P(A)=k)=(k !)^{-1} \nu(A)^{k} \exp [-\nu(A)], k=0,1,2, \cdots .
$$

Conversely, any $\eta$ in $E_{0}$ can be approximated by a sequence of step functions $\eta_{n}=\sum c_{n, j} \chi_{A_{n, j}}$ and

$$
I^{P}\left(\eta_{n}\right)=\sum c_{n, j} \bar{P}\left(A_{n, j}\right) \longrightarrow I^{P}(\eta) \quad \text { in }\left(L_{P}^{2}\right) \text { as } n \rightarrow \infty \text {. }
$$

Moreover, if $\eta_{n}$ converges to $\eta$ which is also in $L^{1}(T, \nu)$, then

$$
\sum c_{n, j} P\left(A_{n, j}\right) \longrightarrow I^{P}(\eta)+\bar{\eta} \quad \text { in }\left(L_{P}^{2}\right) .
$$

Thus, we introduce a notation of stochastic integrals:

$$
\int_{T} \eta(u) d P(u) \equiv I^{P}(\eta)+\bar{\eta} \text { and } \int_{T} \eta(u) d \bar{P}(u) \equiv I^{P}(\eta)
$$

Since $P(A)$ is subjected to a Poisson distribution, $\int_{T} \eta(u) d P(u)$ is a $Z$-valued (a.s.) random variable, if $\eta(t)$ takes its values only in $Z$. This guarantees that the real valued random variable 


$$
\begin{aligned}
f^{P}(\eta ; x) & \equiv \exp \left[\int_{T} \log (1+\eta(u)) d P(u)-\bar{\eta}\right] \\
& =(-1)^{P(\{u ; \eta(u)<-1\})} \exp \left[\int_{T} \log |1+\eta(u)| d P(u)-\bar{\eta}\right]
\end{aligned}
$$

is well defined independently of the choice of branches of $\log (1+\eta(t))$, since $\nu(\{u ; \eta(u)<-1\})<\infty$ for $\eta \in \mathscr{E}$. By (2.13),

$$
\int_{\sigma^{*}} f^{P}(\eta ; x) d \mu_{P}(x)=1
$$

is proved. Obviously, we have the relation

$$
f^{P}(\eta ; x) f^{P}(\zeta: x)=f^{P}(\eta \zeta+\eta+\zeta ; x) \cdot \exp \left[(\eta, \zeta)_{0}\right] \quad \text { a.s. }
$$

Hence we get the equality

$$
\left(f^{P}(\eta ; x), f^{P}(\zeta ; x)\right)_{\left(L_{P}^{2}\right)}=\exp \left[(\eta, \zeta)_{0}\right]=\exp [\langle\eta, \zeta\rangle]
$$

For given $\eta \in E_{0}=L^{2}(T, \nu)$, we can choose a sequence $\left\{\eta_{n}\right\}$ such that $\eta_{n}$ converges to $\eta$ in $E_{0}$ and that $f^{P}\left(\eta_{n} ; x\right)$ is well defined by (2.15). By (2.18), we have that

$$
\begin{aligned}
& \left\|f^{P}\left(\eta_{n}\right)-f^{P}\left(\eta_{m}\right)\right\|_{\left(L_{P}^{2}\right)}=\exp \left[\left\|\eta_{n}\right\|_{0}^{2}\right]+\exp \left[\left\|\eta_{m}\right\|_{0}^{2}\right]-2 \exp \left[\left(\eta_{n}, \eta_{m}\right)_{0}\right] \longrightarrow 0 \\
& \text { as } n, m \rightarrow \infty \text {. }
\end{aligned}
$$

Therefore the non-linear mapping $f^{P}$ can be extended to $L^{2}(T, \nu)$ continuously, and it satisfies the formulae (2.16), (2.17) and (2.18). On the other hand, the expression

$$
f^{P}(\eta ; x)=\exp [\langle x, \log (1+\eta)\rangle-\bar{\eta}]
$$

is possible, if $\eta$ belongs to the set $\mathscr{E} \equiv\{\exp [\zeta(t)]-1 ; \zeta \in \mathscr{E}\}$, which is a total subset of $\mathscr{E}$ by virtue of Assumption [A.2]. Now we define an isomorphism $\mathscr{S}_{P}$ from $\left(L_{P}^{2}\right)$ onto $\mathscr{F}^{(0)}$ by

$$
\left(\mathscr{S}_{P} \varphi\right)(\xi) \equiv \int_{\mathscr{G}^{*}} \varphi(x) f^{P}(\xi ; x) d \mu_{P}(x), \xi \in \mathscr{E}
$$

THEOREM 2.1. The two different $L^{2}$-spaces $\left(L_{G}^{2}\right)$ and $\left(L_{P}^{2}\right)$ are isomorphic to the common functional space $\mathscr{F}^{(0)}$ under the isomorphisms $\mathscr{S}_{G}$ and $\mathscr{S}_{P}$, respectively;

$$
\left(\mathscr{S}_{X} \varphi\right)(\xi)=\int_{\delta^{*}} \varphi(x) f^{X}(\xi ; x) d \mu_{X}(x)=\left(\varphi, f^{X}(\xi)\right)_{\left(L_{X}^{2}\right)}, \quad X=G, P
$$


where $f^{G}$ and $f^{P}$ are defined by (2.4) and (2.19), respectively. In particular, we have that

$$
\left(\mathscr{S}_{X} f^{X}(\eta)\right)(\xi)=\exp [\langle\xi, \eta\rangle], \quad \mathscr{S}_{X} 1=1 .
$$

Proof. Let $\mathscr{\mathscr { H }}_{X}$ be the linear hull of $\left\{f^{x}(\eta) ; \eta \in \mathscr{S}(R)\right\} . \quad$ By (2.5), (21.6) and (2.18), we have (2.21). Therefore $\mathscr{S}_{X}$ is an isomorphism from the closure of $\mathscr{H}_{X}$ onto $\mathscr{F}^{(0)}$, because the equalities

$$
\begin{aligned}
\left(\mathscr{S}_{X} f^{X}(\eta), \mathscr{S}_{X} f^{X}(\zeta)\right)_{\mathscr{F}^{(0)}} & =(\exp [\langle\xi, \eta\rangle], \exp [\langle\xi, \zeta\rangle])_{\mathscr{g}(0)} \\
& =\exp [\langle\eta, \zeta\rangle]=\left(f^{X}(\eta), f^{X}(\zeta)\right)_{\left(L_{X}^{2}\right)}
\end{aligned}
$$

hold for $X=G, P$. Suppose that a $\varphi(x) \in\left(L_{X}^{2}\right)$ is orthogonal to $\mathscr{\mathscr { H }}_{X}$. Then $\left(\mathscr{S}_{X} \varphi\right)(\xi)=\left(f^{X}(\xi), \varphi\right)_{\left(L_{X}^{2}\right)}=0$ for any $\xi \in \mathscr{E}$. By the definition of $f^{X}(\xi ; X)$, we can show that

$$
\int_{6^{*}} \varphi(x) \exp [i\langle x, \xi\rangle] d \mu_{X}(x)=0 \quad \text { for any } \xi \in \mathscr{E} .
$$

This implies that $\varphi(x)=0$. Therefore $\stackrel{\mathscr{H}}{X}_{X}$ is dense in $\left(L_{X}^{2}\right), X=G, P$.

We now see some formulae related to $\mathscr{S}_{P}$. By (2.15), we have

$$
\left(\mathscr{S}_{P} \exp \left[I^{P}(\eta ; \cdot)\right]\right)(\xi)=\exp \left[\int_{T}(\exp [\eta(u)]-1)(\xi(u)+1) d \nu-\bar{\eta}\right]
$$

Following [9], we define Generalized Charlier polynomials $\left\{C_{n}(x ; \eta)\right\}_{n=0}^{\infty}$ of Possion white noise as follows. For $x \in \mathscr{E}^{*}$, there exists a $p>0$ such that $x$ belongs to $E_{-p}$. Then for a given $\eta \in \mathscr{E}, \eta \neq 0, \log (1+\omega \eta(t))$, $|\omega|<1 /\left(C_{p}\|\eta\|_{p}\right)$, are analytic vectors in $E_{p}$ by the Assumption [A.2]. Therefore $\langle x, \log (1+\omega \eta)\rangle$ is analytic in small $\omega$. Hence

$$
\left.C_{n}(x ; \eta) \equiv \frac{d^{n}}{d \omega^{n}} f^{P}(\omega \eta ; x)\right|_{\omega=0}
$$

is defined as a continuous functional of $x$. By the proof of Proposition 3.4 , it will be shown that the derivative in (2.23) exists in the strong sense in $\left(L_{P}^{2}\right)$ and that

$$
\left(\mathscr{S}_{P} C_{n}(x ; \eta)\right)(\xi)=\langle\xi, \eta\rangle^{n}
$$

Applying Proposition 3.4, we have that

$$
\begin{aligned}
\left(C_{n}(x ; \eta), C_{m}(x ; \zeta)\right)_{\left(L_{\boldsymbol{P}}^{\prime}\right)} & =\left(\langle\xi, \eta\rangle^{n},\langle\xi, \zeta\rangle^{m}\right)_{\mathscr{F}(0)} \\
& =\delta_{n, m} n !\langle\eta, \zeta\rangle^{n},
\end{aligned}
$$




$$
f^{P}(\omega \eta ; x)=\sum_{n=0}^{\infty} \frac{\omega^{n}}{n !} C_{n}(x ; \eta) \quad \text { in }\left(L_{P}^{2}\right)
$$

Lastly we derive the recursive formulae of the $C_{n}(x ; \eta)$ 's from the equality

$$
\begin{aligned}
& \frac{d}{d \omega} f^{P}(\omega \eta ; x)=\left\{\left\langle x, \frac{\eta}{1+\omega \eta}\right\rangle-\bar{\eta}\right\} f^{P}(\omega \eta ; x) \quad \text { for small } \omega \\
& \left\{\begin{array}{l}
C_{n+1}(x ; \eta)=\sum_{j=0}^{n}(-1)^{n-j} \frac{n !}{j !}\left\langle x, \eta^{n-\jmath+1}\right\rangle C_{\jmath}(x ; \eta)-\bar{\eta} C_{n}(x ; \eta) \\
C_{0}(x ; \eta)=1, \quad C_{1}(x ; \eta)=\langle x, \eta\rangle-\bar{\eta} .
\end{array}\right.
\end{aligned}
$$

By the same way we define

$$
\begin{aligned}
& C_{n_{1}, \cdots, n_{k}}\left(x ; \eta_{1}, \cdots, \eta_{k}\right) \\
& \left.\quad \equiv \frac{\partial^{n_{1}}}{\partial \omega^{n_{1}}} \cdots \frac{\partial^{n_{k}}}{\partial \omega^{n_{k}}} f^{p}\left(\omega_{1} \eta_{1}+\cdots+\omega_{k} \eta_{k} ; x\right)\right|_{\omega_{1}=0, \cdots, \omega_{k}=0} .
\end{aligned}
$$

Then the following theorem is shown by Lemma 3.5 (cf. [9]).

Theorem 2.2. Let $\left\{\eta_{k}\right\}(\subset \mathscr{S}(\boldsymbol{R}))$ be a c.o.n.s. of $L^{2}(\boldsymbol{R})$. Then $\left\{\left(n_{1} ! \cdots\right.\right.$ $\left.\left.n_{k} !\right)^{-1 / 2} C_{n_{1}, \cdots, n_{k}}\left(x ; \eta_{1}, \cdots, \eta_{k}\right)\right\}$ is a c.o.n.s. of $\left(L_{P}^{2}\right)$.

\section{§3. Gel'fand triplet $\mathscr{F} \subset \mathscr{F}^{(0)} \subset \mathscr{F}^{*}$}

In this section, we will discuss the structure of the Hilbert space $\mathscr{F}^{(0)}$ given in Section 2. The same discussion has been given in [10] mainly in connection with Fock's spaces without details of proofs. Let us begin with a basic triplet $\mathscr{E} \subset E_{0} \equiv L^{2}(T, \nu) \subset \mathscr{E}^{*}$ which satisfies (2.1) and [A.2]. The inclusions $E_{p} \subseteq E_{0} \subseteq E_{-p}, p \geq 1$, mean that every $\eta \in E_{0}$ is an element of the dual space $E_{-p}$ of $E_{p}$ and satisfies

$$
\langle\xi, \eta\rangle=(\xi, \eta)_{0} \quad \text { for any } \xi \in E_{p},
$$

and that there exists an isomorphism $\theta_{p}$ from $E_{-p}$ to $E_{p}$ satisfying

$$
\langle\xi, \zeta\rangle=\left(\xi, \theta_{p} \zeta\right)_{p}=\left(\theta_{p}^{-1} \xi, \zeta\right)_{-p} \quad \text { for } \xi \in E_{p}, \zeta \in E_{-p} .
$$

We write $\theta_{-p} \equiv \theta_{p}^{-1}$. Define a subset $\stackrel{\mathscr{F}}{\mathscr{F}^{(0)}}$ by

$$
\mathscr{\mathscr { F }} \equiv\left\{\sum b_{j} \exp \left[\left\langle\xi, \eta_{j}\right\rangle\right] ; \eta_{j} \in \mathscr{E}, b_{j} \in R\right\}
$$

and introduce inner products $(,)_{\tilde{F}(p)}$ on $\mathscr{\mathscr { F }}$ by

$$
(\exp [\langle\xi, \eta\rangle], \exp [\langle\xi, \zeta\rangle])_{\mathscr{F}(p)}=\exp \left[(\eta, \zeta)_{p}\right]
$$


by the positive definiteness of $\exp \left[(\eta, \zeta)_{p}\right]$. Let $\mathscr{F}^{(p)}$ be the completion of $\mathscr{\mathscr { F }}$ with respect to the inner product $(,)_{\mathscr{F}(p)}$.

Proposition 3.1. $\mathscr{F}^{(p)}$ is a Hilbert space with reproducing kernel $\exp \left[(\xi, \eta)_{-p}\right], \xi, \eta \in \mathscr{E}$, and hence each element $U(\xi)$ of $\mathscr{F}^{(p)}$ is a continuous non-linear functional which can be continuously extended to $E_{-p}$.

Proof. Suppose that $\left\{\zeta_{n}\right\} \subset \mathscr{E}$ and $\left\|\zeta_{n}-\zeta\right\|_{p} \rightarrow 0$ as $n \rightarrow \infty$. Then, it is easily seen that $\exp \left[\left\langle\xi, \zeta_{n}\right\rangle\right]$ is a Cauchy sequence in $\mathscr{F}^{(p)}$, because of the equality

$$
\begin{aligned}
& \left\|\exp \left[\left\langle\xi, \zeta_{n}\right\rangle\right]-\exp \left[\left\langle\xi, \zeta_{m}\right\rangle\right]\right\|_{\mathscr{F}(p)}^{2} \\
& \quad=\exp \left[\left\|\zeta_{n}\right\|_{p}^{2}\right]+\exp \left[\left\|\zeta_{m}\right\|_{p}^{2}\right]-2 \exp \left[\left(\zeta_{n}, \zeta_{m}\right)_{p}\right] .
\end{aligned}
$$

Let $W_{\zeta}$ be the limit, $W_{\zeta}(\xi) \equiv \lim _{n \rightarrow \infty} \exp \left[\left\langle\xi, \zeta_{n}\right\rangle\right]$ in $\mathscr{F}^{(p)}$. Then for $U(\xi)$ $=\sum b_{j} \exp \left[\left\langle\xi, \eta_{j}\right\rangle\right]$ in $\stackrel{\circ}{\mathscr{F}}$, we have equalities

$$
\begin{aligned}
\left(U, W_{\zeta}\right)_{\mathscr{F}(p)} & =\lim _{n \rightarrow \infty}\left(U(\xi), \exp \left[\left\langle\xi, \zeta_{n}\right\rangle\right]\right)_{\mathscr{F}(p)} \\
& =\sum b_{j} \exp \left[\left(\zeta, \eta_{j}\right)_{p}\right]=U\left(\theta_{-p} \zeta\right),
\end{aligned}
$$

which imply $\left(U, W_{\theta_{p} \eta}\right)_{\mathscr{x}(p)}=U(\eta)$ for $\eta \in \mathscr{E}$. Therefore $U_{n}(\eta)=\left(U_{n}, W_{\theta_{p} \eta}\right)_{\mathscr{x}(p)}$ converges to $U(\eta)=\left(U, W_{\theta_{p} \eta}\right)_{\mathscr{F}(p)}$ for each fixed $\eta \in \mathscr{E}$, if $U_{n}(\in \mathscr{\mathscr { F }})$ converges to $U$ in $\mathscr{F}^{(P)}$ as $n \rightarrow \infty$. Since $\theta_{p} \mathscr{E}$ is dense in $E_{p},\left\{W_{\theta_{p} \eta} ; \eta \in \mathscr{E}\right\}$ is a total subset of $\mathscr{F}^{(p)}$. Hence the values $U(\eta), \eta \in \mathscr{E}$, characterize the vector $U$ in $\mathscr{F}^{(p)}$, uniquely. Thus $\mathscr{F}^{(p)}$ can be considered as a functional space on $\mathscr{E}$. Since the equalities

$$
W_{\theta_{p} \zeta}(\eta)=\left(W_{\theta_{p} \zeta}, W_{\theta_{p} \eta}\right)_{\mathcal{F}(p)}=\exp \left[\left\langle\theta_{p} \zeta, \eta\right\rangle\right]=\exp \left[(\zeta, \eta)_{-p}\right]
$$

are easily seen, together with $U(\eta)=\left(U, W_{\theta_{p}}\right)_{\mathscr{F}(p)}$, we can conclude that $W_{\theta_{p} \xi}(\eta)=\exp \left[(\zeta, \eta)_{-p}\right]$ is the reproducing kernel of $\mathscr{F}^{(p)}$.

By $(2.1),(\xi, \eta)_{p+1}-(\xi, \eta)_{p}$ is a positive definite functional on $\mathscr{E}$. Therefore $\exp \left[(\xi, \eta)_{p+1}\right]-\exp \left[(\xi, \eta)_{p}\right]$ is also positive definite and hence

$$
\|U\|_{\mathscr{F}(p+1)} \geq\|U\|_{\mathscr{F}(p)} \quad \text { for } U \in \stackrel{\circ}{\mathscr{F}}
$$

holds. Thus we have natural inclusions

$$
\mathscr{F}^{(p+1)} \subseteq \mathscr{F}^{(p)}, \quad p \in Z
$$

Let $\mathscr{F}$ be the projective limit of $\mathscr{F}^{(p)}$ as $p \rightarrow \infty$, and let $\mathscr{F}^{*}$ be the inductive limit of $\mathscr{F}^{(p)}$ as $p \rightarrow-\infty$. 
Proposition 3.2. Each $\mathscr{F}^{(-p)}$ is the dual of $\mathscr{F}^{(p)}, p \in Z$, and $\mathscr{F}^{*}$ is the dual of $\mathscr{F}$. Moreover

$$
\langle U(\xi), \exp [\langle\xi, \eta\rangle]\rangle=U(\eta) .
$$

Proof. Define a transformation $\Theta_{p}$ on $: \stackrel{\mathscr{F}}{\text { by }}$

$$
\Theta_{p} U(\xi)=\sum b_{j} \exp \left[\left\langle\xi, \theta_{p} \eta_{j}\right\rangle\right] \quad \text { for } U(\xi)=\sum b_{j} \exp \left[\left\langle\xi, \eta_{j}\right\rangle\right] .
$$

Then for $V(\xi)=\sum c_{k} \exp \left[\left\langle\xi, \zeta_{k}\right\rangle\right] \in \stackrel{\circ}{\mathscr{F}}$, it holds that

$$
\begin{aligned}
\left(\Theta_{p} U(\xi), \Theta_{p} V(\xi)\right)_{\mathscr{F}(p)} & =\sum b_{j} c_{k} \exp \left[\left(\theta_{p} \eta_{j}, \theta_{p} \zeta_{k}\right)_{p}\right] \\
& =\sum b_{j} c_{k} \exp \left[\left(\eta_{j}, \zeta_{k}\right)_{-p}\right]=(U(\xi), V(\xi))_{\mathscr{F}(-p)}, \\
\left(\Theta_{p} U(\xi), V(\xi)\right)_{\mathscr{F}^{\prime}(p)}= & \sum b_{j} c_{k} \exp \left[\left(\theta_{p} \eta_{j}, \zeta_{k}\right)_{p}\right] \\
= & \sum b_{j} c_{k} \exp \left[\left\langle\eta_{j}, \zeta_{k}\right\rangle\right]=(U(\xi), V(\xi))_{\mathscr{F}(0)} .
\end{aligned}
$$

Therefore $\Theta_{p}$ can be extended to an isomorphism from $\mathscr{F}^{(-p)}$ to $\mathscr{F}^{(p)}$, because $\Theta_{-p}$ gives the inverse of $\Theta_{p}$. By (3.5), $\mathscr{F}^{(-p)}$ is the dual space of $\mathscr{F}^{(p)}$.

Thus we have a triplet $\mathscr{F} \subset \mathscr{F}^{(0)} \subset \mathscr{F}^{*}$. We will show later that it is a Gel'fand triplet, if so is the basic triplet $\mathscr{E} \subset E_{0} \subset \mathscr{E} *$ (Proposition 3.6). Now we discuss the decomposition of $\mathscr{F}^{(p)}$.

Lemma 3.3. Let $\left\{U_{n}(\xi)\right\}$ be a bounded sequence in $\mathscr{F}^{(p)}$. If the limit, $\lim _{n \rightarrow \infty} U_{n}(\xi)=U(\xi)$, exists for each $\xi \in \mathscr{E}$, then $U(\xi)$ belongs to $\mathscr{F}^{(p)}$ and $U_{n}(\xi)$ converges to $U(\xi)$ weakly in $\mathscr{F}^{(p)}$.

Proof. Since $\mathscr{F}$ is dense in $\mathscr{F}^{(-p)}$, Proposition 3.2 implies the assertion obviously.

Proposition 3.4. For any $\eta, \zeta \in \mathscr{E},\langle\xi, \eta\rangle^{n}$ and $\langle\xi, \zeta\rangle^{m}$ belong to $\mathscr{F}^{(p)}$ and they satisfy the equality

$$
\left.(\langle\xi, \eta\rangle)^{n},\langle\xi, \zeta\rangle^{m}\right)_{\mathscr{F}^{(p)}}=\delta_{n, m} n !(\eta, \zeta)_{p}^{n} .
$$

Let $\mathscr{F}_{n}^{(p)}$ be the subspace of $\mathscr{F}^{(p)}$ spanned by $\left\{\langle\xi, \eta\rangle^{n} ; \eta \in \mathscr{E}\right\}$. Then we have

$$
\mathscr{F}^{(p)}=\sum_{n=0}^{\infty} \oplus \mathscr{F}_{n}^{(p)}
$$

Proof. Obviously,

$$
\lambda^{-n}(\exp [\lambda\langle\xi, \eta\rangle]-1)^{n}=\lambda^{-n} \sum_{k=1}^{n}\left(\begin{array}{l}
n \\
k
\end{array}\right)(-1)^{n-k} \exp [\langle\xi, \lambda k \eta\rangle]
$$


belongs to $\mathscr{F}^{(p)}$ and converges to $\langle\xi, \eta\rangle^{n}$ as $\lambda \rightarrow 0$ for each $\xi \in \mathscr{E}$. Since we have

$$
\begin{aligned}
\left\|\lambda^{-n}\left(e^{\lambda\langle\xi, \eta\rangle}-1\right)^{n}\right\|_{\mathscr{F}^{(p)}}^{2}=\lambda^{-2 n} \sum_{k, j=1}^{n}\left(\begin{array}{l}
n \\
k
\end{array}\right)\left(\begin{array}{l}
n \\
j
\end{array}\right)(-1)^{2 n-k-j} e^{\lambda 2 k j\|\eta\|_{p}^{2}} \\
=\lambda^{-2 n} \sum_{k=1}^{n}\left(\begin{array}{l}
n \\
k
\end{array}\right)(-1)^{n-k}\left(\exp \left[k \lambda^{2}\|\eta\|_{p}^{2}\right]-1\right)^{n} \\
\stackrel{\lambda \rightarrow 0}{\longrightarrow} \sum_{k=0}^{n}\left(\begin{array}{l}
n \\
k
\end{array}\right)(-1)^{n-k} k^{n}\|\eta\|_{p}^{2 n}=n !\|\eta\|_{p}^{2 n},
\end{aligned}
$$

$\langle\xi, \eta\rangle^{n}$ is in $\mathscr{F}^{(p)}$ and its norm is dominated by $(n !)^{1 / 2}\|\eta\|_{p}^{n}$. Therefore

$$
\exp [\omega\langle\xi, \eta\rangle]=\sum_{n=0}^{\infty} \frac{\omega^{n}}{n !}\langle\xi, \eta\rangle^{n}
$$

converges strongly in $\mathscr{F}^{(p)}$. Hence we have

$$
\begin{aligned}
\exp \left[\omega \lambda(\eta, \zeta)_{p}\right] & =(\exp [\omega\langle\xi, \eta\rangle], \exp [\lambda\langle\xi, \zeta\rangle])_{\mathscr{F}(p)} \\
& =\sum_{n, m=0}^{\infty} \frac{\omega^{n} \lambda^{m}}{n ! m !}\left(\langle\xi, \eta\rangle^{n},\langle\xi, \zeta\rangle\right)^{m}{ }_{\mathscr{F}(p)}
\end{aligned}
$$

Thus we have proved the first assertion, which assures the orthogonality of $\mathscr{F}_{n}^{(p)}$ 's. Suppose that $U(\xi) \in \mathscr{F}^{(p)}$ is orthogonal to every $\mathscr{F}_{n}^{(p)}$. By Proposition 3.2 , we have

$$
U(\eta)=\langle U(\xi), \exp [\langle\xi, \eta\rangle]\rangle=\sum_{n=0}^{\infty} \frac{1}{n !}\left(U(\xi),\left\langle\xi, \theta_{p} \eta\right\rangle^{n}\right)_{\mathscr{F}(p)}=0
$$

for any $\eta \in \mathscr{E}$.

LEMMA 3.5.

(i) For $\eta_{j} \in E_{p}, n_{j} \geq 0, j=1,2, \cdots, k, n=n_{1}+n_{2}+\cdots+n_{k}$,

$$
\left\|\prod_{j}\left\langle\xi, \eta_{j}\right\rangle^{n_{j}}\right\|_{\mathcal{F}^{(p)}} \leq(n !)^{1 / 2} \prod_{j}\left\|\eta_{j}\right\|_{p}^{n_{j}}
$$

(ii) If $\left\{\eta_{j}\right\}_{j=1}^{k}$ is an orthogonal system in $E_{p}$, then

$$
\left(\prod_{j}\left\langle\xi, \eta_{j}\right\rangle^{n_{j}}, \prod_{j}\left\langle\xi, \eta_{j}\right\rangle^{m_{j}}\right)_{\mathscr{F}^{(p)}}=\prod_{j}\left[\delta_{n_{j}, m_{j}} n_{j} !\left\|\eta_{j}\right\|_{p}^{2 n_{j}}\right] .
$$

(iii) If $\left\{\eta_{j}\right\}_{j=1}^{\infty}$ is a complete orthonormal system of $E_{p}$, then a collection $\left\{\prod_{j}\left[\left(n_{j} !\right)^{-1 / 2}\left\langle\xi, \eta_{j}\right\rangle^{n_{j}}\right] ; n_{j} \geq 0, n=\sum_{j} n_{j}\right\}$ forms a complete orthonormal system of $\mathscr{F}_{n}^{(p)}$.

Proof. By Proposition 3.4, we have the equalities 


$$
\begin{aligned}
& \left(\left(\sum \omega_{j}\left\langle\xi, \eta_{j}\right\rangle\right)^{n},\left(\sum \lambda_{k}\left\langle\xi, \eta_{k}\right\rangle\right)^{n}\right)_{\mathscr{F}(p)} \\
& \quad=n !\left(\sum \omega_{j} \eta_{j}, \sum \lambda_{k} \eta_{k}\right)_{p}^{n}=n !\left(\sum \omega_{j} \lambda_{k}\left(\eta_{j}, \eta_{k}\right)_{p}\right)^{n},
\end{aligned}
$$

which induce (i). If $\left\{\eta_{j}\right\}$ is an orthogonal system, then the last term of (3.6) is equal to $n !\left(\sum \omega_{j} \lambda_{j}\left\|\eta_{j}\right\|_{p}^{2}\right)^{n}$. Therefore we see (ii). To show (iii), the completeness must be proved. For any $\zeta \in E_{p}$, put $\zeta_{m}=\sum_{j=1}^{m}\left(\zeta, \eta_{j}\right)_{p} \eta_{j}$. Then we can see that $\left\langle\xi, \zeta_{m}\right\rangle^{n}$ converges to $\langle\xi, \zeta\rangle^{n}$ in $\mathscr{F}_{n}^{(p)}$ as $m \rightarrow \infty$. Therefore the system given in (iii) spans $\mathscr{F}_{n}^{(p)}$ by definition.

Proposition 3.6. If $\mathscr{E} \subset E_{0} \subset \mathscr{E}^{*}$ is a Gel'fand triplet satisfying (2.1), then $\mathscr{F} \subset \mathscr{F}^{(0)} \subset \mathscr{F}^{*}$ is also a Gel'fand triplet.

Proof. For given $p$, we can find $q(>p)$ such that the injection ८: $E_{q} \subseteq E_{p}$ is of Hilbert-Schmidt type. Then there exists a complete orthonormal system $\left\{\eta_{j}\right\}$ of $E_{q}$ such that

$$
\left(\eta_{j}, \eta_{k}\right)_{p}=\lambda_{j}^{2} \delta_{j, k}, \quad\|\iota\|_{\text {H.s. }}^{2}=\sum \lambda_{j}^{2}<\infty .
$$

Since $\left|\lambda_{j}\right| \leq \rho<1$ by (2.1), we have that

$$
\begin{aligned}
\sum_{n=0}^{\infty} \sum_{n=n_{1}+n_{2}+\cdots}\left\|\prod_{j}\left(n_{j} !\right)^{-1 / 2}\left\langle\xi, \eta_{j}\right\rangle^{n_{j}}\right\|_{\mathscr{F}^{(p)}}^{2} \\
\quad=\sum_{n=0}^{\infty} \sum_{n=n_{1}+n_{2}+\ldots} \Pi \lambda^{2 n_{j}}=\prod_{j}\left(1-\lambda_{j}^{2}\right)^{-1}<\infty .
\end{aligned}
$$

This shows the assertion.

Lemma 3.7. (i) The series

$$
\sum_{n, m=0}^{\infty} \frac{1}{n ! m !}\langle\xi, \eta\rangle^{n}\langle\xi, \zeta\rangle^{m} \quad \text { and } \quad \sum_{n=0}^{\infty} \frac{1}{n !}\langle\xi, \eta\rangle^{n} \exp [\langle\xi, \zeta\rangle]
$$

converge to the same limit $\exp [\langle\xi, \eta+\zeta\rangle]$ strongly in $\mathscr{F}^{(p)}$ for any $\zeta, \eta \in E_{p}$.

$$
\left\|\exp [\langle\xi, \eta+\zeta\rangle]-\sum_{k=0}^{n} \frac{1}{k !}\langle\xi, \eta\rangle^{k} \exp [\langle\xi, \zeta\rangle]\right\|_{\mathcal{F}^{(p)}}=O\left(\|\eta\|_{p}^{n+1}\right)
$$

(iii) $\quad \mathbb{K}\left\langle\xi, \eta_{1}\right\rangle \cdots\left\langle\xi, \eta_{n}\right\rangle \exp [\langle\xi, \zeta\rangle]\left\|_{\mathscr{F}(p)} \leq \sum_{m=0}^{\infty} \frac{\{(n+m) !\}^{1 / 2}}{m !}\right\| \eta_{1}\left\|_{p} \cdots\right\| \eta_{n}\left\|_{p}\right\| \zeta \|_{p}^{m}$.

Proof. By Lemma 3.5 (i), we have the estimation

$$
\begin{aligned}
& \sum_{n, m} \frac{1}{n ! m !}\left\|\langle\xi, \eta\rangle^{n}\langle\xi, \zeta\rangle^{m}\right\|_{\mathcal{F}(p)} \\
& \quad \leq \sum_{n, m} \frac{\{(n+m) !\}^{1 / 2}}{n ! m !}\|\eta\|_{p}^{n}\|\zeta\|_{p}^{m} \leq 2 \exp \left[2\|\eta\|_{p}^{2}+2\|\zeta\|_{p}^{2}\right]
\end{aligned}
$$


which assures the strong convergences. The assertion (i) follows from Lemma 3.3. The assertions (ii) and (iii) are seen similarly by Lemma 3.3 and Lemma 3.5.

Definition 3.8. Let $U(\xi)$ be a continuous functional on $\mathscr{E}$. If there exist symmetric multilinear functionals $U^{(k)}\left(\xi ; \eta_{1}, \cdots, \eta_{k}\right)$ of $\eta_{1}, \cdots, \eta_{k} \in \mathscr{E}$, satisfying

$$
\begin{aligned}
& U(\xi+\eta)-U(\xi)=\sum_{k=1}^{n} \frac{1}{k !} U^{(k)}(\xi ; \eta, \cdots, \eta)+o\left(\|\eta\|_{p}^{n}\right), \\
& \left|U^{(k)}\left(\xi ; \eta_{1}, \cdots, \eta_{k}\right)\right| \leq c^{(k)}(\xi)\left\|\eta_{1}\right\|_{p} \cdots\left\|\eta_{k}\right\|_{p}, \quad 1 \leq k \leq n,
\end{aligned}
$$

then $U(\xi)$ is said to be $n$-times $E_{p}$-Fréchet differentiable and $U^{(n)}\left(\xi ; \eta_{1}\right.$, $\left.\cdots, \eta_{n}\right)$ is called the $n$-th $E_{p}$-Fréchet derivative of $U(\xi)$ at $\xi$. If $U(\xi)$ is arbitrary many times $E_{p}$-differentiable and the equality

$$
U(\xi+\eta)=\sum_{k=0}^{\infty} \frac{1}{k !} U^{(k)}(\xi ; \eta, \cdots, \eta)
$$

holds with $U^{(0)}(\xi)=U(\xi)$, then $U(\xi)$ is said to be $E_{p}$-Fréchet analytic.

THeOREM 3.9. If $U(\xi)$ is in $\mathscr{F}^{(p)}$, then $U(\xi)$ is $E_{-p}$-Fréchet analytic and the $n$-th $E_{-p}$-derivative is expressed in the form

$$
U^{(n)}\left(\zeta ; \eta_{1}, \cdots, \eta_{n}\right)=\left\langle U(\xi),\left\langle\xi, \eta_{1}\right\rangle \cdots\left\langle\xi, \eta_{n}\right\rangle \exp [\langle\xi, \zeta\rangle]\right\rangle .
$$

Moreover, $U^{(n)}(\zeta ; \cdot)$ can be extended to a symmetric continuous multilinear functional on $E_{-p}$.

Proof. Define $U^{(n)}\left(\zeta ; \eta_{1}, \cdots, \eta_{n}\right)$ by (3.11). Then $U^{(n)}$ 's satisfy (3.8), (3.9) and (3.10) by Lemma 3.7 (ii), (iii) and (i), respectively. The second assertion is obvious by (3.9).

Suppose that $U(\xi)$ is in $\mathscr{F}^{(p)}$. By the theorem, for each $\xi \in \mathscr{E}$ there exists an element $U^{(n)}(\xi ; \cdot)$ belonging to the $n$-fold symmetric tensor product space $E_{p}^{\hat{\otimes} n}$ such that

$$
U^{(n)}\left(\xi ; \eta_{1}, \cdots, \eta_{n}\right)=\left\langle U^{(n)}(\xi ; \cdot), \eta_{1} \hat{\otimes} \cdots \hat{\otimes} \eta_{n}\right\rangle,
$$

where $\eta_{1} \otimes \cdots \otimes \hat{\otimes} \eta_{n}=\varsigma_{n} \eta_{1} \otimes \cdots \otimes \eta_{n}, \eta_{1}, \cdots, \eta_{n} \in E_{-p}$. Here we denote by $\mathfrak{S}_{n}$ the symmetrization on $n$-fold tensor product spaces. We introduce the notation of functional derivative by

$$
\frac{\delta}{\delta \xi\left(t_{1}\right)} \cdots \frac{\delta}{\delta \xi\left(t_{n}\right)} ; U(\xi) \longrightarrow U^{(n)}(\xi ; \cdot) \text {. }
$$


If $p \geq 1$, then $U^{(n)}(\xi ; \cdot)$ is a continuous symmetric function on $T^{n}$ given by

$$
U^{(n)}(\xi ; \cdot)=\frac{\delta}{\delta \xi\left(t_{1}\right)} \cdots \frac{\delta}{\delta \xi\left(t_{n}\right)} U(\xi)=U\left(\xi ; \delta_{t_{1}} \hat{\otimes} \cdots \hat{\otimes} \delta_{t_{n}}\right)
$$

by virtue of Assumption [A.1]. If $p=0$, then $U^{(n)}(\xi ; \cdot)$ belongs to the symmetric $L^{2}$-space $\hat{L}^{2}\left(T^{n}, \nu^{n}\right) \equiv\left\{f_{n} \in L^{2}\left(T^{n}, \nu^{n}\right) ; \Im_{n} f_{n}=f_{n}\right\}$, where $\Im_{n} f_{n}$ is the symmetrization of $f_{n}$ :

$$
\widetilde{S}_{n} f_{n}\left(t_{1}, \cdots, t_{n}\right) \equiv \frac{1}{n !} \sum_{\sigma \in \mathbb{E}_{n}} f\left(t_{\sigma(1)}, \cdots, t_{\sigma(n)}\right)
$$

with the symmetric group $\Im_{n}$ of degree $n$.

THEOREM 3.10. For $U(\xi), V(\xi) \in \mathscr{F}^{(p)}$ and $W(\xi) \in \mathscr{F}^{(-p)}$, we have the following:

$$
U(\eta)=\sum_{n=0}^{\infty} \frac{1}{n !}\left\langle U^{(n)}(0 ; \cdot), \eta^{\hat{\otimes} n}\right\rangle=\sum_{n=0}^{\infty} \frac{1}{n !}\left\langle U(\xi),\langle\xi, \eta\rangle^{n}\right\rangle,
$$

with $U^{(n)}(0 ; \cdot) \in E_{p}^{\hat{\otimes} n}$

$$
\begin{aligned}
& (U(\xi), V(\xi))_{\mathcal{F}^{(p)}}=\sum_{n=0}^{\infty} \frac{1}{n !}\left(U^{(n)}(0 ; \cdot), V^{(n)}(0 ; \cdot)\right)_{E_{p}^{\hat{\otimes} n}}, \\
& \langle U(\xi), W(\xi)\rangle=\sum_{n=0}^{\infty} \frac{1}{n !}\left\langle U^{(n)}(0 ; \cdot), W^{(n)}(0 ; \cdot)\right\rangle .
\end{aligned}
$$

(iv) If, in particular, $p=0$ then

$$
(U(\xi), V(\xi))_{\mathscr{F}^{(0)}}=\sum_{n=0}^{\infty} \frac{1}{n !} \int_{T^{n}} U^{(n)}(0, t) V^{(n)}(0, t) d \nu^{n}(t),
$$

with $\boldsymbol{t}=\left(t_{1}, \cdots, t_{n}\right)$.

Proof. By Theorem 3.9, (3.9) and (3.10) hold for $U(\xi) \in \mathscr{F}^{(p)}$. Applying (3.12), we have the assertion (i). Take $U(\xi)=\exp [\langle\xi, \eta\rangle]$ and $V(\xi)=$ $\exp [\langle\xi, \zeta\rangle]$. Then $U^{(n)}\left(0 ; t_{1}, \cdots, t_{n}\right)=\eta\left(t_{1}\right) \cdots \eta\left(t_{n}\right)=\eta^{\hat{\otimes} n}$ holds by Lemma 3.7 (ii). Therefore we have equalities

$$
\begin{aligned}
(U(\xi), V(\xi))_{\mathscr{F}(p)} & =\exp \left[(\eta, \zeta)_{p}\right]=\sum_{n=0}^{\infty} \frac{1}{n !}\left(\eta^{\hat{\otimes} n}, \zeta^{\hat{\otimes} n}\right)_{E_{p}^{\hat{\otimes}} n} \\
& =\sum_{n=0}^{\infty} \frac{1}{n !}\left(U^{(n)}(0 ; \cdot), V^{(n)}(0 ; \cdot)\right)_{E_{p}^{\hat{\otimes}} n}
\end{aligned}
$$




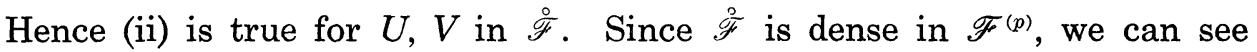
that (ii) is true for $U, V$ in $\mathscr{F}^{(p)}$. (iii) and (iv) are proved similarly.

In the following, we will show that $\left\langle g_{m}, \xi^{\hat{\otimes} m}\right\rangle U(\xi)$ and $\left\langle U^{(m)}(\xi ; \cdot), G_{m}\right\rangle$ belong to $\mathscr{F}^{(p)}$, if $U(\xi) \in \mathscr{F}^{(p+1)}, g_{m} \in E_{p}^{\hat{\otimes}^{m}}$ and $G_{m} \in E_{-p}^{\hat{\otimes} m}$. The help of Fock's space is useful for the proof. The direct sum

$$
\exp \left[\hat{\otimes} E_{p}\right] \equiv \sum_{n=0}^{\infty} \oplus(n !)^{1 / 2} E_{p}^{\hat{\otimes} n}
$$

with inner product

$$
\left(\left(f_{n}\right)_{n \geq 0},\left(g_{n}\right)_{n \geq 0}\right)_{\operatorname{cxp}\left[\hat{\otimes} E_{p}\right]}=\sum_{n=0}^{\infty} n !\left(f_{n}, g_{n}\right)_{E_{p}^{\hat{\otimes}} n}
$$

is called Fock's space. Its dual space is $\exp \left[\hat{\otimes} E_{-p}\right]$ with the canonical bilinear form

$$
\left\langle\left(f_{n}\right)_{n \geq 0},\left(G_{n}\right)_{n \geq 0}\right\rangle=\sum_{n=0}^{\infty} n !\left\langle f_{n}, G_{n}\right\rangle
$$

for $\left(f_{n}\right)_{n \geq 0} \in \exp \left[\hat{\otimes} E_{p}\right],\left(G_{n}\right)_{n \geq 0} \in \exp \left[\hat{\otimes} E_{-p}\right]$. Then we have natural inclusions $E_{p+1}^{\hat{\otimes} n} \subseteq E_{p}^{\hat{\otimes} n}$ and $\exp \left[\hat{\otimes} E_{p+1}\right] \subseteq \exp \left[\hat{\otimes} E_{p}\right]$ for $p \in Z$ by virtue of (2.1). We denote by $\mathscr{E}^{\hat{\otimes}}$ and $\exp [\hat{\otimes} \mathscr{E}]$ then projective limits of $E_{p}^{\hat{\otimes} n}$ and $\exp \left[\hat{\otimes} E_{p}\right]$, respectively. Denote by $\mathscr{E}^{*} \hat{\otimes} n$ and $\exp \left[\hat{\otimes} \mathscr{E}^{*}\right]$ their duals. By Theorem 3.10 and (3.15), we have an isomorphism $\Theta^{\mathscr{F} \rightarrow \mathscr{S}}$ from $\mathscr{F}^{(p)}$ to $\exp \left[\hat{\otimes} E_{p}\right]$;

$$
\Theta^{\xi \rightarrow \varepsilon}: U(\xi) \longrightarrow\left(\frac{1}{n !} U^{(n)}(0 ; \cdot)\right)_{n \geq 0} \text {. }
$$

The inverse of $\Theta^{g \rightarrow C}$ is given by

$$
\Theta^{\varepsilon \rightarrow F}:\left(f_{n}\right)_{n \geq 0} \longrightarrow U(\xi)=\sum_{n=0}^{\infty}\left\langle f_{n}, \xi^{\hat{\otimes} n}\right\rangle .
$$

For example, we can see that

$$
\begin{gathered}
\Theta^{\mathscr{F} \rightarrow \xi}: \exp [\langle\xi, \eta\rangle] \longrightarrow \exp [\hat{\otimes} \eta] \equiv\left(\frac{1}{n !} \eta^{\hat{\otimes} n}\right)_{n \geq 0}, \\
\Theta^{\mathscr{F} \rightarrow \delta}:\left\langle\xi^{\hat{\otimes} m}, g_{m}\right\rangle \exp [\langle\xi, \eta\rangle] \longrightarrow\left(\frac{1}{(n-m) !} g_{m} \hat{\otimes} \eta^{\hat{\otimes}(n-m)}\right)_{n \geq m}
\end{gathered}
$$

for $\eta \in E_{p}$ and $g_{m} \in E_{p}^{\hat{\otimes} m}$.

For $f_{n} \in E_{p}^{\hat{\otimes} n}$ and $g_{m} \in E_{p}^{\hat{\otimes} m}$, we define a symmetric tensor product $f_{n} \hat{\otimes} g_{m}$ by the symmetrization of $f_{n} \otimes g_{m}: f_{n} \hat{\otimes} g_{m} \equiv \widetilde{S}_{n+m}\left(f_{n} \otimes g_{m}\right)$. For $G_{n+m} \in$ $E_{p}^{\hat{\otimes}(n+n)}$, define $f_{n} * G_{n+m}$ as an element of $E_{-p}^{\hat{\otimes} m}$ satisfying 


$$
\left\langle g_{m}, f_{n} * G_{n+m}\right\rangle=\left\langle f_{n} \hat{\otimes} g_{m}, G_{n+m}\right\rangle \quad \text { for any } g_{m} \in E_{p}^{\hat{\otimes} m} .
$$

Then we obviously have the estimations

$$
\begin{aligned}
& \left\|f_{n} \hat{\otimes} g_{m}\right\|_{E_{p}^{\hat{\otimes}}(n+m)} \equiv\left\|\Im_{n+m}\left(f_{n} \otimes g_{m}\right)\right\|_{E_{p}^{\hat{\otimes}(n+m)}} \\
& \quad \leq\left\|f_{n} \otimes g_{m}\right\|_{E_{p}^{\otimes(n+m)}} \leq\left\|f_{n}\right\|_{E_{p}^{\otimes n}}^{\otimes n}\left\|g_{m}\right\|_{E_{p}^{\otimes m}}^{\otimes m}=\left\|f_{n}\right\|_{E_{p}^{\hat{\otimes}} n}\left\|g_{m}\right\|_{E_{p}^{\hat{\otimes}} m}
\end{aligned}
$$

and

$$
\begin{aligned}
\left\|f_{n} * G_{n+m}\right\|_{E_{-p}^{\hat{\otimes}} m} & =\sup _{\left\|g_{m}\right\|_{E_{p}^{\hat{x}} m=1}}\left|\left\langle g_{m}, f_{n} * G_{n+m}\right\rangle\right| \\
& =\sup _{\|\|_{m} \|_{E_{p}^{\hat{\otimes}} m=1}^{\hat{\otimes}}}\left|\left\langle f_{n} \hat{\otimes} g_{m}, G_{n+m}\right\rangle\right| \\
& \leq \sup _{\left\|g_{m}\right\|_{E_{p}^{\hat{\otimes}} m=1}^{\hat{\otimes}}}\left\|f_{n} \hat{\otimes} g_{m}\right\|_{E_{p}^{\hat{\otimes}}(n+m)}\left\|G_{n+m}\right\|_{E_{-p}^{\hat{\otimes}(n+m)}} \\
& \leq\left\|f_{n}\right\|_{E_{p}^{\hat{\otimes}} n}\left\|G_{n+m}\right\|_{E_{-p}^{\hat{\otimes}}(n+m)} .
\end{aligned}
$$

For $\Xi=\left(f_{n}\right)_{n \geq 0} \in \exp \left[\hat{\otimes} E_{p}\right]$, define a projection $\pi_{n}$ to $E_{p}^{\hat{\otimes}_{n}}$ by

$$
\pi_{n}(\Xi) \equiv f_{n}
$$

For $g_{m} \in E_{p}^{\hat{\otimes} m}$ and $G_{m} \in E_{-p}^{\hat{\otimes} m}$, define two operators:

$$
\left\{\begin{array}{l}
a\left(G_{m}\right) \Xi \equiv\left(\frac{(n+m) !}{n !} G_{m} * \pi_{n+m} \Xi\right)_{n \geq 0}, \\
a^{*}\left(g_{m}\right) \Xi \equiv\left(g_{m}^{\hat{\otimes}} \pi_{n-m} \Xi\right)_{n \geq m} .
\end{array}\right.
$$

LEMMA 3.11.

$$
\begin{gathered}
\text { (i ) } \quad\left\|a\left(G_{m}\right) \Xi\right\|_{\exp \left[\hat{\otimes} E_{p}\right]} \leq\left\|G_{m}\right\|_{E_{-p}^{\hat{\otimes}} m}\|\Xi\|_{\exp \left[\hat{\otimes} E_{p+1}\right]} \rho^{m}\left(\left(1-\rho^{2}\right)^{-m-1} m !\right)^{1 / 2}, \\
\text { (ii) }\left\|a^{*}\left(g_{m}\right) \Xi\right\|_{\exp \left[\hat{\otimes} E_{p}\right]} \leq\left\|g_{m}\right\|_{E_{p}^{\hat{\otimes}} m}\|\Xi\|_{\exp \left[\hat{\otimes} E_{p+1}\right]}\left(\left(1-\rho^{2}\right)^{-m-1} m !\right)^{1 / 2} \\
\text { (iii) } \quad \text { for } \Xi \in \exp \left[\hat{\otimes} E_{p+1}\right], \Xi^{\prime} \in \exp \left[\hat{\otimes} E_{-p+1}\right], \\
\left\langle a\left(G_{m}\right) \Xi, \Xi^{\prime}\right\rangle=\left\langle\Xi, a^{*}\left(G_{m}\right) \Xi^{\prime}\right\rangle .
\end{gathered}
$$

Proof. By (2.1), (3.21) and (3.22), we have

$$
\begin{aligned}
& \left\|a\left(G_{m}\right) \Xi\right\|_{\exp \left[\hat{\otimes} E_{p}\right]}^{2}=\sum_{n=0}^{\infty} \frac{((n+m) !)^{2}}{n !}\left\|G_{m} * \pi_{n+m} \Xi\right\|_{E_{p}^{2} \hat{\otimes}_{n}}^{2} \\
& \quad \leq \sum_{n=0}^{\infty} \frac{((n+m) !)^{2}}{n !}\left\|G_{m}\right\|_{E_{-p}^{\hat{\otimes}} n}\left\|\pi_{n+m} \Xi\right\|_{E_{p}^{\hat{\otimes}(n+m)}}^{2} \\
& \quad \leq\left\|G_{m}\right\|_{E_{-p}^{\hat{\otimes}} m}^{2} \sup _{n \geq 0}\left[\frac{(n+m) !}{n !} \rho^{2(n+m)}\right] \sum_{n=0}^{\infty}(n+m) !\left\|\pi_{n+m} \Xi\right\|_{E_{p+1}^{2} \hat{\otimes}(n+m)}^{2},
\end{aligned}
$$

and similarly 


$$
\begin{aligned}
\left\|a^{*}\left(g_{m}\right) \Xi\right\|_{\exp \left[\hat{\otimes} E_{p}\right]}^{2} & =\sum_{n=0}^{\infty}(n+m) !\left\|g_{m} \hat{\otimes} \pi_{n} \Xi\right\|_{E_{p}^{\hat{\otimes}}(n+m)}^{2} \\
& \leq\left\|g_{m}\right\|_{E_{p}^{\hat{\otimes}} m}^{2} \sum_{n=0}^{\infty} \frac{(n+m) !}{n !} \rho^{2 n} n !\left\|\pi_{n} \Xi\right\|_{E_{p+1}^{2} \hat{\otimes}^{n}}^{2 n}
\end{aligned}
$$

Since the estimation

$$
\sup _{n \geq 0} \frac{(n+m) !}{n !} t^{n} \leq \sum_{n \geq m} \frac{n !}{(n-m) !} t^{n-m}=\frac{d^{m}}{d t^{m}}(1-t)^{-1}=m !(1-t)^{-m-1}
$$

holds for $0<t<1$, we have the assertions (i) and (ii). The assertion (iii) is obvious by definition.

Proposition 3.12. Let $\Xi=\left(f_{n}\right)_{n \geq 0}$ be in $\exp \left[\hat{\otimes} E_{p+1}\right]$ and put $U(\xi) \equiv$ $\langle\Xi, \exp [\hat{\otimes} \xi]\rangle$. Then for $G_{m} \in E_{-p}^{\hat{\otimes} m}$ and $g_{m} \in E_{p}^{\hat{\otimes} m}$, it holds that

$$
\begin{aligned}
& \left\langle a\left(G_{m}\right) \Xi, \exp [\hat{\otimes} \xi]\right\rangle=\left\langle U^{(m)}(\xi ; \cdot), G_{m}(\cdot)\right\rangle, \\
& \left\langle a^{*}\left(g_{m}\right) \Xi, \exp [\hat{\otimes} \xi]\right\rangle=\left\langle\xi^{\hat{\otimes} m}, g_{m}\right\rangle U(\xi) .
\end{aligned}
$$

Proof. By (3.16), (3.21) and (3.24), we have

$$
\begin{aligned}
\langle a(G) \Xi, \exp [\hat{\otimes} \xi]\rangle & =\sum_{n=0}^{\infty} \frac{(n+m) !}{n !}\left\langle G_{m} * f_{n+m}, \xi^{\hat{\otimes} n}\right\rangle \\
& =\sum_{n=0}^{\infty} \frac{(n+m) !}{n !}\left\langle f_{n+m}, G_{m} \hat{\otimes} \xi^{\hat{\otimes} n}\right\rangle .
\end{aligned}
$$

On the other hand, for $G_{m}=\eta_{1} \hat{\otimes} \cdots \hat{\otimes} \eta_{m}$

$$
\begin{aligned}
U^{(m)}\left(\zeta ; G_{m}\right) & =\left\langle U(\xi),\left\langle\xi, \eta_{1}\right\rangle \cdots\left\langle\xi, \eta_{n}\right\rangle \exp [\langle\xi, \zeta\rangle]\right\rangle \\
& =\sum_{n=m}^{\infty} \frac{1}{(n-m) !}\left\langle f_{n}, G_{m} \hat{\otimes} \zeta^{\hat{\otimes}(n-m)}\right\rangle
\end{aligned}
$$

holds by Theorem 3.9, (3.18) and (3.20). Therefore the equality (3.26) holds for $G_{m}$ in a dense subset of $E_{-p}^{\hat{\otimes} m}$, which is spanned by linear combinations of $\eta_{1} \hat{\otimes} \cdots \hat{\otimes} \eta_{m}$. By Theorem 3.9 and Lemma 3.10 (i), we can show (3.26) for any $G_{m}$. The proof of (3.27) is similar.

TheOREM 3.13. For $U(\xi) \in \mathscr{F}^{(p+1)}, g_{m} \in E_{p}^{\hat{\otimes} m}$ and $G_{m} \in E_{-p}^{\hat{\otimes} m},\left\langle\xi^{\hat{\otimes} m}, g_{m}\right\rangle U(\xi)$ and $\left\langle U^{(m)}(\xi ; \cdot), G_{m}\right\rangle$ are in $\mathscr{F}^{(p)}$, and moreover their norms are estimated as

$$
\begin{aligned}
& \left\|\left\langle\xi^{\hat{\otimes} m}, g_{m}\right\rangle U(\xi)\right\|_{\mathscr{F}^{(p)}} \leq\left\|g_{m}\right\|_{E_{p}^{\hat{\otimes}} m}\|U\|_{\left.\mathscr{F}^{(p+1}\right)}\left(\left(1-\rho^{2}\right)^{-m-1} m !\right)^{1 / 2} \\
& \left.\mathbb{\|} U^{(m)}(\xi ; \cdot), G_{m}\right\rangle\left\|_{\mathscr{F}^{(p)}} \leq\right\| G_{m}\left\|_{E_{-p}^{\hat{\otimes} m}}\right\| U \|_{\left.\mathcal{F}^{(p+1}\right)} \rho^{m}\left(\left(1-\rho^{2}\right)^{-m-1} m !\right)^{1 / 2}
\end{aligned}
$$


Further, for $V(\xi) \in \mathscr{F}^{(-p-1)}$,

$$
\left\langle\left\langle\xi^{\hat{\otimes} m}, g_{m}\right\rangle U(\xi), V(\xi)\right\rangle=\left\langle U(\xi),\left\langle V^{(m)}(\xi ; \cdot), g_{m}(\cdot)\right\rangle\right\rangle \text {. }
$$

Proof is obvious by Lemma 3.10 and Proposition 3.12 .

CoRollary 3.14. For $p \geq 1, \frac{\delta}{\delta \xi\left(t_{1}\right)} \cdots \frac{\delta}{\delta \xi\left(t_{n}\right)}$ is a continuous operator from $\mathscr{F}^{(p)}$ to $\mathscr{F}^{(p+1)}$ and it depends on $t=\left(t_{1}, \cdots, t_{n}\right)$, continuously. The multiplication

$$
\xi(t) \cdot: U(\xi) \longrightarrow \xi(t) U(\xi)
$$

is a continuous operator from $\mathscr{F}^{(p)}$ to $\mathscr{F}^{(p-1)}$ and it depends on $t$ continuously, whenever $p<0$. Moreover, $\xi(t)$. is the dual of the operator $\frac{\delta}{\delta \xi(t)}$.

For arbitrary $p \in Z, \frac{\delta}{\delta \xi(t)}$ and $\xi(t)$. have meanings as operator valued generalized functions;

$$
\begin{aligned}
& \left\langle\eta, \frac{\delta}{\delta \xi(\cdot)}\right\rangle U(\xi) \equiv\left\langle U^{(1)}(\xi ; \cdot), \eta\right\rangle=U^{(1)}(\xi ; \eta), \\
& \langle\eta, \xi(\cdot)\rangle U(\xi) \equiv\langle\eta, \xi\rangle U(\xi) .
\end{aligned}
$$

For $U(\xi)$ in $\mathscr{F}$ (or $\left.\mathscr{F}^{*}\right), V(\xi) \equiv \xi(s) U(\xi)$ belongs to $\mathscr{F}^{*}$. Then the equality

$$
V^{(1)}(\xi ; \eta)=\xi(s) U^{(1)}(\xi ; \eta)+\left\langle\delta_{s}, \eta\right\rangle U(\xi)
$$

holds. Hence

$$
\left.\frac{\delta}{\delta \xi(t)}(\xi(s) U(\xi))=\xi(s) \frac{\delta}{\delta \xi(t)} U(\xi)+\delta_{s}^{\prime} t\right) U(\xi)
$$

holds as operator valued generalized functions. In this sense, we have commutation relations, which are called the canonical commutation relations;

$$
\begin{aligned}
& {\left[\frac{\delta}{\delta \xi(t)}, \xi(s)\right]=\delta_{s}(t)} \\
& {\left[\frac{\delta}{\delta \xi(t)}, \frac{\delta}{\delta \xi(s)}\right]=0 \text { and }[\xi(t), \xi(s)]=0}
\end{aligned}
$$




\section{$\S 4$. Calculus on white noises}

We put the assumptions [A.1], [A.2] and [A.3] together with the condition (2.1) on norms. In Theorem 2.1, we have seen that $L^{2}\left(\mathscr{E}^{*}, \mu_{X}\right)$ is isomorphic to $\mathscr{F}^{(0)}$ under the isomorphism $\mathscr{S}_{X}$ given by (2.20) for $X=G$, $P$. In Section 3, we have established a Gel'fand triplet $\mathscr{F} \subset \mathscr{F}^{(0)} \subset \mathscr{F}^{*}$. For $p \geq 0$, put $\mathscr{H}_{X}^{(p)} \equiv \mathscr{S}_{X}^{-1} \mathscr{F}^{(p)}$ and induce inner product $(,) \mathscr{H}_{X}^{(p)}$ from $\mathscr{F}^{(p)}$; that is,

$$
(\varphi, \psi)_{\mathscr{H}_{X}^{(p)}} \equiv\left(\mathscr{S}_{X} \varphi, \mathscr{S}_{X} \psi\right)_{\mathscr{F}(p)}
$$

for $X=G, P$. Let $\mathscr{H}_{X}$ be the projective limit of $\mathscr{H}_{X}^{(p)}$ as $p \rightarrow \infty$. Obviously, we have inclusions $\mathscr{H}_{X}^{(p+1)} \subseteq \mathscr{H}_{X}^{(p)}, p \geq 0$. Then $\mathscr{H}_{X}^{(p)}$ is isomorphic to $\mathscr{F}^{(p)}$ and $\mathscr{H}_{X}$ is isomorphic to $\mathscr{F}$ under the common transformation $\mathscr{S}_{X}$, which can be expressed by

$$
\left(\mathscr{S}_{X} \varphi\right)(\xi)=\left\langle\varphi, f^{x}(\xi ; x)\right\rangle \quad \text { for } \xi \in \mathscr{E},
$$

where $f^{G}(\xi ; x)$ is given by $(2.4)$ and $f^{P}(\xi, x)$ is by (2.15). Since

$$
\left\|f^{X}(\eta ; x)\right\|_{\mathscr{H}_{X}^{(p)}}^{2}=\left\|\left(\mathscr{S}_{X} f^{X}(\eta)\right)(\xi)\right\|_{\mathscr{F}^{(p)}}^{2}=\|\exp [\langle\xi, \eta\rangle]\|_{\mathscr{F}^{(p)}}^{2}=\exp \left[\|\eta\|_{p}^{2}\right]
$$

for any $p \geq 0, f^{X}(\eta, x)$ belongs to $\mathscr{H}_{X}=\bigcap_{p>0} \mathscr{H}_{X}^{(p)}$. Now let $\mathscr{H}_{X}^{(-p)}$ be the dual of $\mathscr{H}_{X}^{(p)}$ and let $\mathscr{H}_{X}^{*}$ be the dual of $\mathscr{H}_{X}$.

Proposition 4.1. The transformation $\mathscr{S}_{X}$ given by (4.2) defines an isomorphism from $\mathscr{H}_{X}^{(p)}$ to $\mathscr{F}^{(p)}$ for any $p \in Z$. In particular, the element 1 in $\mathscr{H}_{X}^{(p)}$ is transformed to 1 in $\mathscr{F}^{(p)}$ by it.

Proof. By definition, the assertion is true for $p \geq 0$. Let us discuss the case of $p<0$. Notice that the linear subset $\mathscr{\mathscr { H }}_{X} \equiv\left\{\sum_{j} b_{j} f^{X}\left(\eta_{j} ; x\right) ; \eta_{j}\right.$ $\left.\in \mathscr{E}, b_{j} \in \boldsymbol{R}\right\}$ of $\mathscr{H}_{X}$ is dense in $\mathscr{H}_{X}^{(p)}$. For $\varphi(x)=\sum b_{j} f^{X}\left(\eta_{j} ; x\right) \in \mathscr{H}_{X}$, put $U(\xi) \equiv\left(\mathscr{S}_{X} \varphi\right)(\xi)=\sum b_{j} \exp \left[\left\langle\xi, \eta_{j}\right\rangle\right]$. Then we have

$$
\begin{aligned}
\|\varphi\|_{\mathscr{P}_{X}^{(p)}} & =\sup _{\|\psi\|_{\left.\mathscr{f}_{X}^{\prime}-p\right)=1}}|\langle\varphi, \psi\rangle|=\sup _{\|\psi\|_{\left.\mathscr{f}_{X}^{\prime}-p\right)=1}}\left|(\varphi, \psi)_{0}\right| \\
& =\sup _{\left.\|V(\xi)\| \xi^{(}-p\right)}|\langle U(\xi), V(\xi)\rangle|=\|U(\xi)\|_{\mathscr{F}^{(p)}} .
\end{aligned}
$$

Since $\mathscr{\mathscr { H }}_{X}$ is dense in $\mathscr{H}_{X}$ and since $\left(\mathscr{S}_{X} \varphi\right)(\xi)$ is continuous in $\varphi$. for each fixed $\xi \in \mathscr{E}$, we can conclude that $\mathscr{S}_{X}$ is isomorphic. Obviously,

$$
\left(\mathscr{S}_{X} 1\right)(\xi)=\int_{\delta^{*}} f^{X}(\xi ; x) d \mu_{X}=1 .
$$

For $g_{m} \in E_{p}^{\hat{\otimes} m}$ and $G_{m} \in E_{-p}^{\hat{\otimes} m}$, define operators $A^{*}\left(g_{m}\right)$ and $A\left(G_{m}\right)$ by 


$$
\left\{\begin{array}{l}
A^{*}\left(g_{m}\right) \varphi \equiv \mathscr{S}_{X}^{-1}\left(\left\langle\xi^{\hat{\otimes} m}, g_{m}\right\rangle\left(\mathscr{S}_{X} \varphi\right)(\xi)\right) \\
A\left(G_{m}\right) \varphi \equiv \mathscr{S}_{X}^{-1}\left(\left\langle\left(\mathscr{S}_{X} \varphi\right)^{(m)}(\xi ; \cdot), G_{m}\right\rangle\right) .
\end{array}\right.
$$

Then Theorem 3.13 implies the following theorem immediately:

THEOREM 4.2. (i) For $\varphi \in \mathscr{H}_{X}^{(p+1)}, g \in E_{p}^{\hat{\otimes} m}$ and $G_{m} \in E_{-p}^{\hat{\otimes} m}, A^{*}\left(g_{m}\right) \varphi$ and $A\left(G_{m}\right) \varphi$ belong to $\mathscr{H}_{X}^{(p)}$, and moreover their norms are estimated as follows;

$$
\begin{aligned}
& \left\|A^{*}\left(g_{m}\right) \varphi\right\|_{\mathscr{X}_{X}^{(p)}} \leq\left\|g_{m}\right\|_{E_{p}^{\hat{\otimes}} m}\|\varphi\|_{\mathscr{X}_{X}^{(p+1}}\left(\left(1-\rho^{2}\right)^{-m-1} m !\right)^{1 / 2}, \\
& \left\|A\left(G_{m}\right) \varphi\right\|_{\mathscr{F}_{X}^{(p)}} \leq\left\|G_{m}\right\|_{E} \hat{\otimes}_{-p}^{\hat{\otimes} m}\|\varphi\|_{\mathscr{F}_{X}^{(p+1)}} \rho^{m}\left(\left(1-\rho^{2}\right)^{-m-1} m !\right)^{1 / 2} .
\end{aligned}
$$

(ii) For $g_{m} \in \mathscr{E}^{\mathscr{\otimes} m}=\bigcap_{p>0} E_{p}^{\hat{\otimes} m}$ and $G_{m} \in \mathscr{E}^{* \otimes \hat{\otimes} m}=\bigcup_{p<0} E_{p}^{\hat{\otimes} m}, A^{*}\left(g_{m}\right)$ and $A\left(G_{m}\right)$ are continuous operators on $\mathscr{H}_{X}$. Further $A^{*}\left(G_{m}\right)$ and $A\left(g_{m}\right)$ are continuous operators on $\mathscr{H}_{X}^{*}$ for $X=G, P$.

(iii) $A^{*}\left(g_{m}\right)$ (resp. $A^{*}\left(G_{m}\right)$ ) is the dual operator of $A\left(g_{m}\right)$ (resp. of $\left.A\left(G_{m}\right)\right)$.

THEOREM 4.3. For $\varphi$ in $\mathscr{H}_{X}^{(p)}$, there exists an element $\left(f_{n}\right)_{n \geq 0}$ of $\exp \left[\hat{\otimes} E_{p}\right]$ such that

$$
\varphi=\sum_{n=0}^{\infty} A^{*}\left(f_{n}\right) 1, \quad\left(\mathscr{S}_{X} \varphi\right)(\xi)=\sum_{n=0}^{\infty}\left\langle\xi^{\hat{\otimes} n}, f_{n}\right\rangle \text { and }\left\|\varphi^{2}\right\|_{\mathscr{F}_{X}^{(p)}}=\sum_{n=0}^{\infty} n !\left\|f_{n}\right\|_{E_{p}^{\otimes} \hat{\otimes} n} .
$$

Proof. Since $\mathscr{S}_{X}$ is an isomorphism from $\mathscr{H}_{X}^{(p)}$ to $\mathscr{F}^{(p)}, \mathscr{S}_{X} \varphi$ belongs to $\mathscr{F}^{(p)}$, if $\varphi \in \mathscr{H}_{X}^{(p)}$. Put $f_{n} \equiv \frac{1}{n !}\left(\mathscr{S}_{X} \varphi\right)^{(n)}(0 ; \cdot)$. Then $\left(f_{n}\right)_{n \geq 0}$ belongs to $\exp \left[\hat{\otimes} E_{p}\right]$ and $\left(\mathscr{S}_{X} \varphi\right)(\xi)=\sum\left\langle\xi^{\hat{\otimes} n}, f_{n}\right\rangle$ holds by (3.17) and (3.18). By the definition of $A^{*}\left(f_{n}\right)$ in (4.3) and Proposition 4.1, we have

$$
\sum_{n=0}^{\infty} A^{*}\left(f_{n}\right) 1=\sum_{n=0}^{\infty} \mathscr{S}_{X}^{-1}\left(\left\langle\xi^{\hat{\otimes} n}, f_{n}\right\rangle\right)=\varphi .
$$

The last equality of the theorem comes from Theorem 3.10 (iv).

In the following, it is convenient to define $A^{*}\left(g_{m}\right)$ and $A\left(g_{m}\right)$ even for $g_{m} \in E_{p}^{\otimes_{m}}$ by $A^{*}\left(g_{m}\right)=A^{*}\left(\Im_{m} g_{m}\right)$ and $A\left(g_{m}\right)=A\left(\Im_{m} g_{m}\right)$. As an special case, observe $\delta_{t}$ in $E_{-1}$. Then we write, for simplicity

$$
\left\{\begin{array}{l}
\partial_{t} \equiv A\left(\delta_{t}\right)=\mathscr{S}_{X}^{-1} \frac{\delta}{\delta \xi(t)} \mathscr{S}_{X}, \\
\partial_{t}^{*} \equiv A^{*}\left(\delta_{t}\right)=\mathscr{S}_{X}^{-1} \xi(t) \cdot \mathscr{S}_{X},
\end{array}\right.
$$

and we use the notation of multi-indices;

$$
\partial_{t} \equiv \partial_{t_{1}} \cdots \partial_{t_{m}} \quad \text { and } \quad \partial_{t}^{*} \equiv \partial_{t_{1}}^{*} \cdots \partial_{t_{m}}^{*}
$$


for $t=\left(t_{1}, \cdots, t_{m}\right) \in T^{m}$. By Theorem 4.2, estimations

$$
\begin{aligned}
& \left\|\partial_{t} \varphi-\partial_{s} \varphi\right\|_{\mathscr{F}_{X}^{(p)}} \leq\left\|\delta_{t}-\delta_{s}\right\|_{-1}\|\varphi\|_{\mathscr{F}_{X}^{(p+1)}}\left(1-\rho^{2}\right)^{-1} \rho^{-p-1}, \\
& \left\|\partial_{t}^{*} \varphi-\partial_{s}^{*} \varphi\right\|_{\left.\mathscr{F}_{X}^{(}-p\right)} \leq\left\|\delta_{t}-\delta_{s}\right\|_{-1}\|\varphi\|_{\left.\mathscr{P}_{X}^{(}-p+1\right)}\left(1-\rho^{2}\right)^{-1},
\end{aligned}
$$

are obtained, for $p \geq 1$; that is, $\partial_{t}$ and $\partial_{t}^{*}$ depend on $t$ continuously in $\mathscr{H}_{X}$ and $\mathscr{H}_{X}^{*}$, respectively, by virtue of Assumption [A.1].

Proposition 4.4. For $g_{m} \in \mathscr{E}^{\hat{\otimes} m}$ and $\varphi \in \mathscr{H}_{X}$,

$$
A\left(g_{m}\right) \varphi=\int_{T^{m}} d \nu^{m}(t) g_{m}(t) \partial_{t} \varphi
$$

holds in $\mathscr{H}_{X}$. For $g_{m} \in E_{0}^{\hat{\otimes} m}=\hat{L}^{2}\left(T^{m}, \nu^{m}\right)$ and $\psi \in \mathscr{H}_{X}^{*}$,

$$
A^{*}\left(g_{m}\right) \psi=\int_{T^{m}} d \nu^{m}(t) g_{m}(t) \partial_{t}^{*} \psi
$$

holds in $\mathscr{H}_{X}^{*}$. Here integrals are understood as Bochner integrals.

Proof. By Theorem 4.2 (i) and by (2.2), the existence of the Bochner integrals are obvious. Since $\mathscr{S}_{X}$ is an isomorphism, we have

$$
\begin{gathered}
\left(\mathscr{S}_{X} \int_{T^{m}} d \nu^{m}(t) g_{m}(t) \partial_{t} \varphi\right)(\xi)=\int_{T^{m}} d \nu^{m} g_{m} \frac{\delta}{\delta \xi\left(t_{1}\right)} \cdots \frac{\delta}{\delta \xi\left(t_{m}\right)}\left(\mathscr{S}_{X} \varphi\right)(\xi) \\
=\left\langle g_{m}(\cdot),\left(\mathscr{S}_{X} \varphi\right)^{(m)}(\xi ; \cdot)\right\rangle=\left(\mathscr{S}_{X}\left(A\left(g_{m}\right) \psi\right)\right)(\xi)
\end{gathered}
$$

and

$$
\begin{gathered}
\left(\mathscr{S}_{X} \int_{T^{m}} d \nu^{m} g_{m} \partial_{t}^{*} \psi\right)(\xi)=\int_{T^{m}} d \nu^{m} g_{m} \xi\left(t_{1}\right) \cdots \xi\left(t_{m}\right)\left(\mathscr{S}_{X} \psi\right)(\xi) \\
=\left\langle\xi^{\hat{\otimes} m}, g_{m}\right\rangle\left(\mathscr{S}_{X} \psi\right)(\xi)=\left(\mathscr{S}_{X}\left(A^{*}\left(g_{m}\right) \psi\right)\right)(\xi) .
\end{gathered}
$$

Thus we have the assertions.

For the calculus of Gaussian white noise, operators $A(\cdot)$ and $A^{*}(\cdot)$ are very powerful tools as seen in [11]. For the calculus of Poisson white noise, we need more complicated operators. Let $f_{n+k+m}$ be in $\mathscr{E}^{\hat{\otimes}(n+k+m)}$ and put

$$
A_{n, k, m}\left(f_{n+k+m}\right) \equiv \int_{T^{n+k+m}} d \nu^{n}(\boldsymbol{t}) d \nu^{k}(\boldsymbol{s}) d \nu^{m}(\boldsymbol{u}) f_{n+k+m}(\boldsymbol{t}, \boldsymbol{s}, \boldsymbol{u}) \partial_{\boldsymbol{t}}^{*} \partial_{\boldsymbol{g}}^{*} \partial_{\boldsymbol{g}} \partial_{\boldsymbol{u}}
$$

Let $g_{j}$ be in $\mathscr{E}^{\hat{\otimes} \mathfrak{j}}$. Put

$$
f_{n+k+m} \hat{\otimes}_{m, k} g_{j}(t, s, r) \equiv \widetilde{S}_{n+j-m} \int_{T^{m}} d \nu^{m}(\boldsymbol{u}) f_{n}(\boldsymbol{t}, \boldsymbol{s}, \boldsymbol{u}) g_{j}(\boldsymbol{r}, \boldsymbol{s}, \boldsymbol{u})
$$


with $\boldsymbol{t}=\left(t_{1}, \cdots, t_{n}\right), \boldsymbol{s}=\left(s_{1}, \cdots, s_{k}\right), \boldsymbol{u}=\left(u_{1}, \cdots, u_{m}\right)$ and $\boldsymbol{r}=\left(r_{1}, \cdots, r_{j-k-m}\right)$ for $k+m<j$. If $k+m \geq j$, then put $f_{n+k+m} \hat{\otimes}_{m, k} g_{j} \equiv 0$. Then we have an estimate

$$
\left\|f_{n+k+m} \hat{\otimes}_{m, k} g_{j}\right\|_{E_{p}^{\hat{X}}(n+j-m)} \leq C_{p}^{k-1}\|\delta\|^{2 m} \rho^{2 m(p-1)}\left\|f_{n+k+m}\right\|_{E_{p}^{\hat{\mathbf{X}}}(n+k+m)}\left\|g_{j}\right\|_{E_{p}^{\hat{\otimes}} j}
$$

for $p \geq 1$, here $C_{p}$ is the constant in the assumption [A.2].

Proposition 4.5. For $f_{n+k+m} \in \mathscr{E}^{\mathscr{\otimes} \hat{\otimes}(n+k+m)}$ and $g_{j} \in \mathscr{E}^{\hat{\mathrm{\Delta}} j}$, we have the following assertions;

(i)

$$
\begin{aligned}
& A_{n, k, m}\left(f_{n+k+m}\right) A^{*}\left(g_{j}\right) 1=\frac{j !}{(j-m-k) !} A^{*}\left(f_{n+k+m} \hat{\otimes}_{m, k} g_{j}\right) 1, \\
& \left\|A_{n, k, m}\left(f_{n+k+m}\right) A^{*}\left(g_{j}\right) 1\right\|_{\mathscr{P}_{X}^{(p)}} \\
& \quad \leq \frac{j !((n+j-m) !)^{1 / 2}}{(j-m-k) !}\|\delta\|^{2 m} \rho^{2 m(p-1)}\left\|f_{n+k+m}\right\|_{E_{p}^{\hat{\otimes}(n+k+m)}\left\|g_{j}\right\|_{E_{p}^{\hat{\otimes} j}} C_{p}^{k-1},}
\end{aligned}
$$

(ii) for $\varphi \in \mathscr{H}_{X}$,

$$
\begin{aligned}
& \left\|A_{n, k, m}\left(f_{n+k+m}\right) \varphi\right\|_{\mathscr{H}_{X}^{(p)}} \leq\left\|f_{n+k+m}\right\|_{E_{p}^{\hat{\otimes}}(n+k+m)}\|\varphi\|_{\left.\mathscr{H}_{X}^{(p)}+q\right)}\|\delta\|^{2 m} \rho^{2 m(p-1)} C_{p}^{k-1} \\
& \left.\quad \times(n+k) !(m+k) !\left(1-\rho^{2 q}\right)^{-n-m-2}\right)^{1 / 2} \rho^{2 q(m+k)} .
\end{aligned}
$$

Proof. (i) Since $\left(\mathscr{S}_{X} A^{*}\left(g_{j}\right) 1\right)(\xi)=\left\langle\xi^{\otimes} j, g_{j}\right\rangle$, it holds that

$$
\mathscr{P}_{X}\left(\partial_{\boldsymbol{u}} A^{*}\left(g_{j}\right) 1\right)(\xi)=\frac{j !}{(j-i) !} \int_{T^{j-i}} g_{j}(\boldsymbol{v}, \boldsymbol{u}) \xi\left(v_{1}\right) \cdots \xi\left(v_{j-i}\right) d \nu^{j-i}(\boldsymbol{v}) .
$$

Hence we have the equality. The estimation follows from (4.7).

(ii) By the results in (i) and Theorem 4.3,

$$
\begin{aligned}
& \left\|A_{n, k, m}\left(f_{n+k+m}\right) \varphi_{\mathscr{H}^{\prime}}\right\|_{\mathscr{H}_{X}^{(p)}}^{2}=\sum_{j \geq m+k} \frac{j !}{(j-m-k) !} A^{*}\left(f_{n+k+m} \hat{\otimes}_{m, k} g_{j}\right) 1 \|_{\mathscr{H}_{X}^{(p)}}^{2} \\
& \leq \sum_{j \geq m+k} \frac{(j !)^{2}(n+j-m) !}{((j-m-k) !)^{2}} C_{p}^{2 k-2}\|\delta\|^{4 m} \rho^{4 m(p-1)}\left\|f_{n+k+m}\right\|_{E_{p}^{\hat{x}}(n+k+m)}^{2}\left\|g_{j}\right\|_{E_{\boldsymbol{p}}^{\hat{\hat{x}}} j}^{\hat{x}_{j}} \\
& \leq \sup _{j \geq m+k} \frac{j !(n+j-m) !}{((j-m-k) !)^{2}} C_{p}^{2 k-2} \rho^{4 q j}\|\delta\|^{4 m} \rho^{4 m(p-1)}\left\|f_{n+k+m}\right\|\left\|_{E_{p}^{\hat{\otimes}}(n+k+m)}^{2}\right\| \varphi \|_{\mathcal{R}_{X}^{(p+q)}}^{2} \text {. }
\end{aligned}
$$

The proof is completed by the estimation

$$
\sup _{k \geq 0} \frac{(j+n) !(j+m) !}{(j !)^{2}} z^{2 j} \leq n ! m !(1-z)^{-m-n-2} \quad \text { for } 0<z<1,
$$

which is shown similarly to (3.25). 
For the convenience in later sections, we discuss a little more. Let $p$ be a natural number. Then by Assumptions [A.1], [A.3] and (2.1), we can choose a natural number $q=q(p)(>p)$ such that the injection $\iota_{p, q}$ from $E_{q}$ into $E_{p}$ is of Hilbert-Schmidt type with

$$
C_{p}\left\|\iota_{p, q}\right\|_{\text {H.s. }} \leq \rho \text {. }
$$

LEMMA 4.6. Let $n_{1}, \cdots, n_{k}$ be natural numbers and let $f_{n}$ be an element of $E_{q}^{\hat{\otimes} n}$ with $n=n_{1}+\cdots+n_{k}$. Then

$$
f_{k}(\boldsymbol{t})=\left\langle\delta_{t_{1}}^{\hat{\otimes} n_{1}} \hat{\otimes} \cdots \hat{\otimes} \delta_{t_{k}}^{\hat{\otimes} n_{k}}, f_{n}\right\rangle
$$

belongs to $E_{p}^{\hat{\otimes} k}$ and its norm is estimated by

$$
\left\|f_{k}\right\|_{E_{p}^{\hat{\otimes}} k} \leq \rho^{n}\left\|f_{n}\right\|_{E_{\boldsymbol{q}}^{\hat{\otimes} n}}
$$

Proof. Let $\left\{e_{j}\right\}$ be a complete orthonormal system of $E_{q}$. Then $f_{n}$ can be expanded as

$$
f_{n}=\sum_{j_{1}, \cdots, j_{n}} c_{j_{1}, \cdots, j_{n}} e_{j_{1}}\left(t_{1}\right) \cdots e_{j_{n}}\left(t_{n}\right) \quad \text { in } E_{q}^{\hat{\otimes} n} .
$$

By Assumption [A.2],

$$
\begin{aligned}
\left\|f_{k}\right\|_{E_{p}^{\hat{\otimes} k}} & \leq \sum_{j_{1}, \cdots, j_{n}} C_{p}^{n-k}\left|c_{j_{1}, \cdots, j_{n}}\right|\left\|e_{j_{1}}\right\|_{p} \cdots\left\|e_{j_{n}}\right\|_{p} \\
& \leq C_{p}^{n-k}\left[\sum\left|C_{j_{1}, \cdots, j_{n}}\right|^{2}\right]^{1 / 2}\left[\sum\left\|e_{j_{1}}\right\|_{p}^{2} \cdots\left\|e_{j_{n}}\right\|_{p}^{2}\right]^{1 / 2} \\
& \leq C_{p}^{n-k}\left\|f_{n}\right\|_{E_{q}^{\hat{\otimes}} n}\left\|\iota_{p, q}\right\|_{\mathrm{H} . \mathrm{S} .}^{n} \leq \rho^{n}\left\|f_{n}\right\|_{E_{q}^{\hat{\otimes}} n} .
\end{aligned}
$$

Let $\alpha=\left\{A_{j}\right\}$ be a countable Borel partition of $T$ such that $0<\nu\left(A_{j}\right)$ $<\infty$. Let $\alpha^{(n)}$ be the collection of subsets $C$ 's such that $C=A_{j_{1}} \times \cdots$ $\times A_{j_{n}}$ with $A_{j_{k}} \in \alpha, A_{j_{k}} \cap A_{j_{i}}=\varnothing$, if $k \neq i$. Put

$$
f_{n}^{(\alpha)}(\boldsymbol{t}) \equiv \sum_{C \in \alpha^{(n)}} \frac{1}{\nu^{n}(C)} \int_{C} f_{n}(\boldsymbol{u}) d \nu^{n}(\boldsymbol{u}) \cdot \chi_{C}(\boldsymbol{t})
$$

for $f_{n} \in L^{2}\left(T^{n}, \nu^{n}\right)$. Then

$$
f_{n}=\lim _{\alpha \uparrow} f_{n}^{(\alpha)} \quad \text { a.s. }
$$

holds, here $\alpha \uparrow$ means the refinement to the partition into the individual points. Multiple Wiener integrals are defined as follows. For $C=A_{j_{1}} \times$ $\cdots \times A_{j_{n}} \in \alpha^{(n)}$, define

$$
I_{n}^{X}\left(\chi_{C}\right) \equiv \prod_{k=1}^{n} I^{X}\left(\chi_{A_{j_{k}}}\right) \quad \text { for } X=G, P .
$$


For $f_{n} \in L^{2}\left(T^{n}, \nu^{n}\right)$, put

$$
I_{n}^{X}\left(f_{n}^{(\alpha)}\right) \equiv \sum_{C \in \alpha^{(n)}} \frac{1}{\nu^{n}(C)} \int_{C} f_{n} d \nu^{n} I_{n}^{X}\left(\chi_{C}\right)
$$

Then we can see that

$$
\left\|I_{n}^{X}\left(f_{n}^{(\alpha)}\right)\right\|_{\mathscr{P}^{(0)}}^{2}=n !\left\|\widetilde{S}_{n} f_{n}^{(\alpha)}\right\|_{L^{2}\left(T^{n}, \nu^{n}\right)}^{2} .
$$

Therefore, we see that $I_{n}^{X}\left(f_{n}^{(\alpha)}\right)$ converges in $\mathscr{H}^{(0)}$ as $\alpha \uparrow$. Define multiple Wiener integrals $I_{n}^{X}\left(f_{n}\right)$, for $X=G, P$, by

$$
I_{n}^{X}\left(f_{n}\right) \equiv \lim _{\alpha \uparrow} I_{n}^{X}\left(f_{n}^{(\alpha)}\right) .
$$

TheOREM 4.7. For $f_{n} \in L^{2}\left(T^{n}, \nu^{n}\right), g_{m} \in L^{2}\left(T^{m}, \nu^{m}\right)$, it holds that

$$
I_{n}^{X}\left(f_{n}\right)=A^{*}\left(f_{n}\right) 1=\int_{T^{n}} d \nu^{n}(\boldsymbol{t}) f_{n}(\boldsymbol{t}) \partial_{t}^{*} 1=I_{n}^{X}\left(\Im_{n} f_{n}\right)
$$

in $\mathscr{H}_{X}^{*}$, and that

$$
\begin{aligned}
\int I_{n}^{X}\left(f_{n}\right) I_{m}^{X}\left(g_{m}\right) d \mu_{X} & =\left(A^{*}\left(f_{n}\right) 1, A^{*}\left(g_{m}\right) 1\right)_{\mathscr{*}_{X}^{(0)}} \\
& =\delta_{n, m} n !\left(\Im_{n} f_{n}, \Im_{m} g_{m}\right)_{\hat{L}^{2}\left(T^{n}, \nu n\right)} .
\end{aligned}
$$

Proof. Let $\alpha=\left\{A_{j}\right\}$ be a partition as in above. Since we have

$$
\begin{gathered}
\left(\mathscr{S}_{P} \exp \left[\sum_{j=1}^{N} I^{P} \lambda_{i}\left(\chi_{A_{j}}\right)\right]\right)(\xi)=\exp \left[\int \xi(t)\left(\exp \left[\sum_{j=1}^{N} \lambda_{j} \chi_{A_{j}}(t)\right]-1\right) d \nu\right] \\
=\exp \left[\sum_{j=1}^{N}\left(\exp \left[\lambda_{j}\right]-1\right) \int \chi_{A_{j}} \xi(t) d \nu(t)\right]
\end{gathered}
$$

for any $N$ by virtue of (2.13),

$$
\begin{aligned}
\left(\mathscr{S}_{P} I_{n}^{P}\left(\chi_{C}\right)\right)(\xi) & =\left(\mathscr{S}_{P} \prod_{k=1}^{n} I^{P}\left(\chi_{A_{j_{k}}}\right)\right)(\xi)=\prod_{k=1}^{n} \int_{A_{j_{k}}} \xi(t) d \nu(t) \\
& =\prod_{k=1}^{n}\left\langle\xi, \chi_{A_{j_{k}}}\right\rangle=\left\langle\xi^{\hat{\otimes}}, \Im_{n} \chi_{C}\right\rangle
\end{aligned}
$$

is obtained for $C=A_{j_{1}} \times \cdots \times A_{j_{n}}$. In the case of $X=G$, we have similarly that

$$
\left(\mathscr{S}_{G} \exp \left[\sum_{j=1}^{N} \lambda_{j} I^{G}\left(\chi_{A_{j}}\right)\right]\right)(\xi)=\exp \left[\left\langle\xi, \sum_{j=1}^{N} \lambda_{j} \chi_{A_{j}}\right\rangle\right]
$$

and hence that

$$
\left(\mathscr{S}_{G} I_{n}^{G}\left(\chi_{C}\right)\right)(\xi)=\left(\mathscr{S}_{G} \prod_{k=1}^{n} I_{n}^{G}\left(\chi_{A_{j k}}\right)\right)(\xi)=\prod_{k=1}^{n}\left\langle\xi, \chi_{A_{j_{k}}}\right\rangle=\left\langle\xi^{\hat{\otimes} n}, \widetilde{S}_{n} \chi_{C}\right\rangle .
$$


Thus for both cases, we have

$$
\left(\mathscr{S}_{X} I_{n}^{X}\left(f_{n}^{(\alpha)}\right)\right)(\xi)=\int_{T^{n}} f_{n}^{(\alpha)}(t) \xi\left(t_{1}\right) \cdots \xi\left(t_{n}\right) d \nu^{n}(\boldsymbol{t})=\left\langle\xi^{\hat{\otimes} n}, \widetilde{S}_{n} f_{n}^{(\alpha)}\right\rangle
$$

which implies that $I_{n}^{X}\left(f_{n}^{(\alpha)}\right)=I_{n}^{X}\left(\widetilde{S}_{n} f_{n}^{(\alpha)}\right)=A^{*}\left(\widetilde{S}_{n} f_{n}^{(\alpha)}\right) 1$ in $\mathscr{H}_{X}^{(0)} . \quad$ By (4.9) and (4.10), we can see that

$$
\begin{aligned}
\left(\mathscr{S}_{X} I_{n}^{X}\left(f_{n}\right)\right)(\xi) & =\lim _{\alpha \uparrow}\left(\mathscr{S}_{X} I_{n}^{X}\left(f_{n}^{(\alpha)}\right)(\xi)=\lim _{\alpha \uparrow}\left\langle\xi^{\hat{\otimes} n}, \widetilde{S}_{n} f_{n}^{(\alpha)}\right\rangle\right. \\
& =\left\langle\xi^{\hat{\otimes} n}, \widetilde{S}_{n} f_{n}\right\rangle .
\end{aligned}
$$

Hence $I_{n}^{X}\left(f_{n}\right)=I_{n}^{X}\left(\Im_{n} f_{n}\right)=A^{*}\left(\Im_{n} f_{n}\right) 1$. By Theorem 3.10, (3.20) and (4.11), we get

$$
\begin{aligned}
\left(I_{n}^{X}\left(f_{n}\right), I_{m}^{X}\left(g_{m}\right)\right)_{\mathscr{X}(0)} & =\left(\left\langle\xi^{\hat{\otimes} n}, \mathfrak{S}_{n} f_{n}\right\rangle,\left\langle\xi^{\hat{\otimes} m}, \mathfrak{S}_{m} g_{m}\right\rangle\right)_{\mathscr{F}(0)} \\
& =\delta_{n, m} n !\left(\mathfrak{S}_{n} f_{n}, \mathfrak{S}_{m} g_{m}\right)_{E_{0}^{\hat{\otimes} n}}
\end{aligned}
$$

Theorem 4.8. The both spaces $\left(L_{X}^{2}\right), X=G, P$, are isomorphic to $\mathscr{F}^{(0)}$ and to $\exp \left[\hat{\otimes} E_{0}\right]$ as is shown in the following diagram:

$$
U(\xi)=\left(\mathscr{S}_{x \varphi}\right)(\xi) / \frac{f_{n}=\frac{1}{n !} U^{(n)}(0 ; t)}{U(\xi)=\sum\left\langle\xi^{\hat{\otimes}}, f_{n}\right\rangle}\left(f_{n}^{2}\right)_{n \geq 0} \in \exp \left[\hat{\otimes} E_{0}\right] .
$$

The diagram shows that both $L^{2}$-spaces $\left(L_{G}^{2}\right)$ and $\left(L_{P}^{2}\right)$ have the same structure connecting with $\mathscr{F}^{(0)}$, the Fock's space and multiple Wiener integrals. Are there any differences between these spaces? In the next section, we will see the multiplication has different expressions in the two cases.

\section{$\S 5$. Multiplication and characterization of white noises}

In $L^{2}\left(\mathscr{E}^{*}, \mu_{X}\right), X=P, G$, the multiplication $(\varphi \cdot \psi)(x)=\varphi(x) \cdot \psi(x)$ has meaning if $\varphi$ or $\psi$ belongs to $L^{\infty}\left(\mathscr{E}^{*}, \mu_{X}\right)$. How can we treat the multiplication in our formulation? In the case of Gaussian, the operator

$$
x^{G}(t) \cdot=\partial_{t}^{*}+\partial_{t}
$$

describes multiplication (see [11]). Actually, 


$$
\langle x, \eta\rangle \varphi=\int_{T} d \nu(t) \eta(t) x_{G}(t) \cdot \varphi \quad \text { in } \mathscr{H}_{G}^{*}
$$

holds for $\varphi \in \mathscr{H}_{G}$ and $\eta \in \mathscr{E}$. One of the authors ([9]) has shown that

$$
x^{P}(t) \cdot=\left(\partial_{t}^{*}+1\right)\left(\partial_{t}+1\right)
$$

describes multiplication for Poisson white noise. More precisely,

$$
\langle x, \eta\rangle \varphi=\int_{T} d \nu(t) \eta(t) x^{P}(t) \cdot \varphi \quad \text { in } \mathscr{H}_{P}^{*}
$$

holds for $\varphi$ in $\mathscr{H}_{P}$ and $\eta$ in $\mathscr{E}$. The idea of (5.2) and (5.4) can be stated as follows. For $\varphi$ in $\mathscr{H}_{X}$, put $U(\xi)=\left(\mathscr{S}_{X} \varphi\right)(\xi)$. By (2.4) and (2.17), we have

$$
\begin{aligned}
\left(\mathscr{S}_{X}\left(f^{x}(\eta ; x) \varphi(x)\right)(\xi)=\int_{\delta^{*}} f^{x}(\xi ; x) f^{x}(\eta ; x) \varphi(x) d \mu_{X}\right. \\
\quad= \begin{cases}U(\xi+\eta) \exp [\langle\xi, \eta\rangle] & \text { for } X=G, \\
U(\xi+\eta+\xi \eta) \exp [\langle\xi, \eta\rangle] & \text { for } X=P .\end{cases}
\end{aligned}
$$

Substitute $\eta$ with $\omega \eta$ and differentiate them at $\omega=0$. Then we have

$$
\begin{aligned}
\mathscr{S}_{G}(\langle x, \eta\rangle \varphi(x))(\xi) & =\langle x, \xi\rangle U(\xi)+U^{(1)}(\xi ; \eta) \\
& =\int_{T} d \nu(t) \eta(t)\left(\xi(t)+\frac{\delta}{\delta \xi(t)}\right) U(\xi)
\end{aligned}
$$

and

$$
\begin{aligned}
\mathscr{S}_{P}(\langle x, \eta\rangle \varphi(x))(\xi) & =(\langle\xi, \eta\rangle+\bar{\eta}) U(\xi)+U^{(1)}(\xi ;(1+\xi) \eta) \\
& =\int_{T} d \nu(t) \eta(t)(1+\xi(t))\left(1+\frac{\delta}{\delta \xi(t)}\right) U(\xi)
\end{aligned}
$$

by (2.4) and (2.17). By (4.4) and by Proposition 4.4, we have (5.2) and (5.4).

To complete the proof of (5.2) (5.4), now we must guarantee that $f^{P}(\eta ; x) \varphi(x)$ belongs at least to $\mathscr{H}_{x}^{*}$ and that the differentiation has meaning in $\mathscr{H}_{X}^{*}$. Here we discuss for the Poisson case. If $\varphi$ is in $\mathscr{H}_{P}$, then $f^{P}(\eta) \varphi$ belongs to $\mathscr{\mathscr { H }}_{P}\left(\subset \mathscr{H}_{P}\right)$ by (2.17) and then (5.5) holds. Actually,

$$
\mathscr{S}_{P}\left(f^{P}(\omega \eta ; x) f^{P}(\xi ; x)\right)(\xi)=\exp [\langle\xi, \omega \eta \zeta+\omega \eta+\zeta\rangle+\omega\langle\eta, \zeta\rangle]
$$

holds. Hence, from the definition (3.3), it follows that

$$
\begin{aligned}
& \varlimsup_{\omega \rightarrow 0}\left\|\frac{1}{\omega}\left(f^{P}(\omega \eta ; x)-1\right) f^{P}(\zeta ; x)\right\|_{\mathscr{C}_{P}^{(p)}}^{2} \\
& \quad=\left\{\|\eta(\zeta+1)\|_{p}^{2}+\left((\zeta, \eta \zeta+\eta)_{p}+\langle\eta, \zeta\rangle\right)^{2}\right\} e^{\|\zeta\|_{p}^{2}} .
\end{aligned}
$$


Thus the derivative of (5.7) at $\omega=0$ exists in the strong sense in $\mathscr{F}$, since the derivative exists for each $\xi \in \mathscr{E}$ and since $\mathscr{F}$ is a nuclear space by Proposition 3.6. Thus $\langle x, \eta\rangle \varphi$ belongs to $\mathscr{H}_{P}$ and (5.6) holds for $\varphi \in \mathscr{H}_{P}$. Similarly we can see for the Gaussian case. Remember that

$$
A(\eta)=\int_{T} d \nu(t) \eta(t) \partial_{t} \quad \text { and } \quad A^{*}(\eta)=\int_{T} d \nu(t) \eta(t) \partial_{t}^{*}
$$

are continuous operators on $\mathscr{H}_{P}$ by Proposition 4.4 and Theorem 4.2 (ii). By Proposition 4.5,

$$
A_{0,1,0}(\eta)=\int_{T} d \nu(s) \eta(s) \partial_{s}^{*} \partial_{s}
$$

is a continuous operator on $\mathscr{H}_{P}$. Therefore

$$
\begin{gathered}
\int_{T} d \nu(t) \eta(t) x^{P}(t)=A_{0,1,0}(\eta)+A^{*}(\eta)+A(\eta)+1 \\
\left(\operatorname{resp} . \int_{T} d \nu(t) \eta(t) x^{G}(t) \cdot=A^{*}(\eta)+A(\eta)\right)
\end{gathered}
$$

defines a continuous operator on $\mathscr{H}_{P}$ (resp. on $\mathscr{H}_{G}$ ). For a given $\varphi \in \mathscr{H}_{X}$, there exists an approximating sequence $\left\{\varphi_{n}\right\} \subset \mathscr{\mathscr { H }}_{X}$ such that $\varphi_{n} \rightarrow \varphi$ in $\mathscr{H}_{X}$ and that

$$
\sum_{n=1}^{\infty}\left\|\varphi_{n}-\varphi\right\|_{\mathscr{P}_{X}^{(0)}}<\infty
$$

Then we see that $\varphi_{n}(x)$ conveges to $\varphi_{n}(x)$ (a.s. $\mu_{X}$ ), and hence that $\lim _{n \rightarrow \infty}\langle x, \eta\rangle \varphi_{n}(x)=\langle x, \eta\rangle \varphi(x)$ (a.s. $\mu_{X}$ ). On the other hand, the continuity of $A(\eta), A^{*}(\eta)$ and $A_{0,1,0}(\eta)$ implies that

$$
\lim _{n \rightarrow \infty}\langle x, \eta\rangle \varphi_{n}=\int_{T} d \nu(t) \eta(t) x^{X}(t) \cdot \varphi \quad \text { in } \mathscr{H}_{X}, X=P, G .
$$

Thus we have completed the proof.

TheOREM 5.1. For $\varphi \in \mathscr{H}_{X},\langle x, \eta\rangle \varphi$ belongs to $\mathscr{H}_{X}$ and

$$
\langle x, \eta\rangle \varphi=\int_{T} d \nu(t) \eta(t) x^{X}(t) \cdot \varphi
$$

holds. The operator $\langle x, \eta\rangle \cdot: \varphi \rightarrow\langle x, \eta\rangle \varphi$ is continuous in $\mathscr{H}_{X}$. Moreover $\langle x, \eta\rangle \varphi \rightarrow x^{X}(t) \cdot \varphi$ in $\mathscr{H}_{X}^{*}$ as $\eta \rightarrow \delta_{t}$ in $E_{-1}$ with $\eta \in \mathscr{E}, \bar{\eta}=1$.

Proof. By Theorem 4.2 and by similar discussion to the proof of Proposition 4.5, we see 


$$
\begin{aligned}
& \|A(\eta) \varphi\|_{\mathscr{P}^{(-1)}} \leq\|\eta\|_{-1}\|\varphi\|_{\mathscr{C}^{(2)}} \rho\left(1-\rho^{2}\right)^{-1} \\
& \left\|A^{*}(\eta) \varphi\right\|_{\mathscr{C}^{(-1)}} \leq\|\eta\|_{-1}\|\varphi\|_{\left.\mathscr{P}^{(0)}\right)} \rho\left(1-\rho^{2}\right)^{-1} \text { and } \\
& \left\|A_{0,1 ; 0}(\eta) \varphi\right\|_{\mathscr{C}^{(-1)}} \leq\|\eta\|_{-1}\|\varphi\|_{\mathscr{P}^{(q+1)}}
\end{aligned}
$$

for $\eta \in \mathscr{E}, \varphi \in \mathscr{H}$ with $q=q(1)$ satisfying (4.8). These estimates imply the last assertion which completes the proof.

For $f \in E_{-p}$ and $\eta \in E_{p}$, multiplication $\eta f$ is defined by

$$
\langle\eta f, \zeta\rangle=\langle f, \eta \zeta\rangle \quad \text { for } \zeta \in E_{p} .
$$

Then the norm of $\eta f$ is evaluated as

$$
\|\eta f\|_{-p}=\sup _{\|\zeta\|_{p}=1}\|\langle f, \eta \zeta\rangle\| \leq\|f\|_{-p}\|\eta \zeta\|_{p} \leq C_{p}\|f\|_{-p}\|\zeta\|_{p} .
$$

This observation show that $A_{0,1,0}(\eta)$ acts on $\mathscr{H}_{X}^{*}$, continuously, too. Thus we have:

\section{Proposition 5.2.}

$$
\begin{gathered}
\langle x, \eta\rangle \cdot=A_{0,1,0}(\eta)+A^{*}(\eta)+A(\eta)+1 \\
\left(\operatorname{resp.}\langle x, \eta\rangle \cdot=A^{*}(\eta)+A(\eta)\right)
\end{gathered}
$$

acts on $\mathscr{H}_{P}^{*}$ (resp. on $\left.\mathscr{H}_{G}^{*}\right)$ continuously, and it is self-dual; that is,

$$
\langle\langle x, \eta\rangle \cdot \varphi, \psi\rangle=\langle\varphi,\langle x, \eta\rangle \cdot \psi\rangle \quad \text { for } \varphi \in \mathscr{H}_{X}, \psi \in \mathscr{H}_{X}^{*} .
$$

Now we show that the multiplication operators $x^{G}(t) \cdot=\partial_{t}^{*}+\partial_{t}$ and $x^{P}(t) \cdot=\left(\partial_{t}^{*}+1\right)\left(\partial_{t}+1\right)$ characterize the measures of white noises.

Let $\mu$ be a probability measure on $\mathscr{E}^{*}$. Suppose that there exists an isomorphism $\mathscr{S}$ from $L^{2}\left(\mathscr{E}^{*}, \mu\right)$ onto $\mathscr{F}^{(0)}$. Then put $\mathscr{H}=\mathscr{S}^{-1} \mathscr{F}$ and induce the topology from $\mathscr{F}$. Let $\mathscr{H}^{*}$ be the dual of $\mathscr{H}$, then $\mathscr{S}$ can be continuously extended to $\mathscr{H}^{*}$ in such a way that $\mathscr{H}^{*}$ is isomorphic to $\mathscr{F}^{*}$ under $\mathscr{S}$. Put $\mathscr{H}^{(p)} \equiv \mathscr{S}^{-1} \mathscr{F}^{(p)}$ and give it the topology induced from $\mathscr{F}^{(p)}$. Operators $\partial_{t}, \partial_{t}^{*}, t \in T$, are defined by

$$
\partial_{t} \equiv \mathscr{S}^{-1} \frac{\delta}{\delta \xi(t)} \mathscr{S}, \quad \partial_{t}^{*} \equiv \mathscr{S}^{-1} \xi(t) \cdot \mathscr{S} .
$$

Operators $A\left(f_{n}\right)$ and $A^{*}\left(f_{n}\right)$ on $\mathscr{H}$ (or on $\mathscr{H}^{*}$ ) can be defined by (4.3). For $f_{n} \in L^{2}\left(T^{n}, \nu^{n}\right)$, the multiple Wiener integral $I_{n}\left(f_{n}\right)$ is defined by

$$
I_{n}\left(f_{n}\right) \equiv A^{*}\left(f_{n}\right) 1 \quad \text { (cf. Theorem 4.7.). }
$$

THEOREM 5.3. Let $\mu$ be a probability measure on $\mathscr{E}^{*}$. Suppose that ther 
exists an isomorphism $\mathscr{S}$ form $L^{2}\left(\mathscr{E}^{*}, \mu\right)$ to $\mathscr{F}^{(0)}$ satisfying

(i) $\mathscr{S} 1=1$,

(ii) $x(t) \cdot=\partial_{t}+\partial_{t}^{*}$ on $\mathscr{H} \equiv \mathscr{S}^{-1} \mathscr{F}$;

that is, $\langle x, \eta\rangle \varphi=\int_{T} d \nu(t) \eta(t) x(t) \cdot \varphi$ holds for $\varphi \in \mathscr{H}$. Then $\mu$ is the measure of Gaussian white noise.

Proof. Let $\varphi$ be in $\mathscr{H}$ and put $U(\xi)=\left(\mathscr{S}_{\varphi}\right)(\xi)$. Then

$$
\mathscr{S}(\langle x, \eta\rangle \varphi)(\xi)=\langle\xi, \eta\rangle U(\xi)+U^{(1)}(\xi ; \eta)
$$

holds by the assumption (ii) and by Theorem 3.13. Therefore $\langle x, \eta\rangle \varphi \in \mathscr{H}$. By the assumption, 1 belongs to $\mathscr{H}$, and hence it is recursively shown that $\langle x, \eta\rangle^{n} \in \mathscr{H} \subset L^{2}\left(\mathscr{E}^{*}, \mu\right)$. Thus any polynomials of $\langle x, \eta\rangle$ belongs to $\mathscr{H}$. Remark that the Hermite's polynomials defined by (2.6) satisfy the additive formula

$$
\left\{\begin{array}{l}
H_{n}(z ; \gamma)-n \gamma H_{n-1}(z ; \gamma)=H_{n+1}(z ; \gamma) \\
H_{0}(z ; \gamma)=1 \text { and } H_{1}(z ; \gamma)=z
\end{array}\right.
$$

Of course, $\left.H_{n}\left(\langle x, \eta\rangle ;\|\eta\|_{0}^{2}\right)\right)$ belongs to $\mathscr{H}$. Put

$$
U_{n}(\xi) \equiv \mathscr{S}\left(H_{n}\left(\langle x, \eta\rangle ;\|\eta\|_{0}^{2}\right)\right)(\xi),
$$

then $U_{0}(\xi)=1$ and $U_{0}^{(1)}(\xi ; t)=0$ hold by (i) and (5.10). By (5.9), $U_{1}(\xi)=$ $\langle\xi, \eta\rangle$ is obtained. Assume that $U_{k}(\xi)=\langle\xi, \eta\rangle^{k}$ for $k \leq n$. Then by (5.10) and (5.9).

$$
\begin{aligned}
U_{n+1}(\xi) & =\mathscr{S}\left(\langle x, \eta\rangle H_{n}\left(\langle x, \eta\rangle ;\|\eta\|_{0}^{2}\right)\right)(\xi)-n\|\eta\|_{0}^{2} U_{n-1}(\xi) \\
& =\langle\xi, \eta\rangle U_{n}(\xi)+\int_{T} d \nu(t) \eta(t) \frac{\delta}{\delta \xi(t)}\langle\xi, \eta\rangle^{n}-n\|\eta\|_{0}^{2}\langle\xi, \eta\rangle^{n-1} \\
& =\langle\xi, \eta\rangle^{n+1} .
\end{aligned}
$$

By Proposition 3.4, $\left\{U_{n}\right\}$ is an orthogonal system with $\left(U_{n}, U_{m}\right)_{\mathscr{F}(p)}=$ $\delta_{n, m} n !\|\xi\|_{p}^{2 n}$. Therefore

$$
\exp [\langle\xi, \eta\rangle]=\sum_{n=0}^{\infty} \frac{1}{n !} U_{n}(\xi)=\sum_{n=0}^{\infty} \mathscr{S}\left(H_{n}\left(\langle x, \eta\rangle ;\|\eta\|_{0}^{2}\right)\right)(\xi)
$$

converges in $\mathscr{F}$. Since $\mathscr{S}$ is an isomorphism, the series

$$
\sum_{n=0}^{\infty} \frac{1}{n !} H_{n}\left(\langle x, \eta\rangle ;\|\eta\|_{0}^{2}\right)=\exp \left[\langle x, \eta\rangle-\frac{1}{2}\|\eta\|_{0}^{2}\right]
$$

obtained by (2.6) converges in $\mathscr{H}$. Hence it holds that 


$$
\begin{aligned}
\int_{\sigma^{*}} \exp \left[\langle x, \eta\rangle-\frac{1}{2}\|\eta\|^{2}\right] d \mu & =\left(\exp \left[\langle x, \eta\rangle-\frac{1}{2}\|\eta\|_{0}^{2}\right], 1\right)_{\mathscr{F}^{(0)}} \\
& =\langle\exp [\langle\xi, \eta\rangle], 1\rangle_{\mathscr{F}^{(0)}}=1 .
\end{aligned}
$$

This implies that

$$
\int_{\Omega^{*}} \exp [i\langle x, \eta\rangle] d \mu=\exp \left[-\frac{1}{2}\|\eta\|_{0}^{2}\right],
$$

and hence $\mu=\mu_{G}$.

THEOREM 5.4. Let $\mu$ be a probability measure on $\mathscr{E} *$ whose support is included in $E_{-r}$ with $r>0$. Suppose that there exists an isomorphism $\mathscr{S}$ from $L^{2}\left(\mathscr{E}^{*}, \mu\right)$ to $\mathscr{F}^{(0)}$ satisfying

(i) $\mathscr{S} 1=1$,

(ii) $x(t) \cdot=\left(\partial_{t}^{*}+1\right)\left(\partial_{t}+1\right)$ on $\mathscr{H}=\mathscr{S}^{-1} \mathscr{F}$.

Then $\mu$ is the measure of Poisson white noise.

Proof. The Charlier polynomials $C_{n}(x ; \eta)$ can be defined by $(2.23)$ as continuous functionals of $x$ and $\eta$, irrespectively of measures on $\mathscr{E}^{*}$. We now show the equality

$$
\mathscr{S}\left(C_{n}(x, \eta)\right)(\xi)=\langle\xi, \eta\rangle^{n} ;
$$

that is, $C_{n}(x ; \eta)=A^{*}\left(\eta^{\hat{\otimes} n}\right) 1=I_{n}\left(\eta^{\hat{\otimes} n}\right)$. If $\varphi$ is in $\mathscr{H}$, then $(\mathscr{S} \varphi)(\xi)$ is in $\mathscr{F}$ and

$$
(\mathscr{S}(\langle x, \zeta\rangle \varphi))(\xi)=\int_{T} d \nu(t) \zeta(t)(1+\xi(t))\left(1+\frac{\delta}{\delta \xi(t)}\right)(\mathscr{S} \varphi)(\xi)
$$

belongs to $\mathscr{F}$. Therefore $\langle x, \zeta\rangle \varphi$ is in $\mathscr{H}$. By (2.27) and by the condition (ii), we have

$$
\left(\mathscr{S} C_{0}(x ; \eta)\right)(\xi)=\mathscr{S} 1=1 .
$$

Assume that $C_{k}(x ; \eta)$ is in $\mathscr{H}$ and $\left(\mathscr{S} C_{k}(x ; \eta)\right)(\xi)=\langle\xi, \eta\rangle^{k}$ holds for $0 \leq k$ $\leq n$. Then applying (5.12),

$$
\begin{aligned}
& \left(\mathscr{S}\left(\left\langle x, \eta^{n-j+1}\right\rangle C_{j}(x ; \eta)\right)\right)(\xi) \\
& \quad=j\left\{\left\langle\xi, \eta^{n-j+2}\right\rangle+\overline{\eta^{n-j+2}}\right\}\langle\xi, \eta\rangle^{j-1}+\left\{\left\langle\xi, \eta^{n-j+1}\right\rangle+\overline{\eta^{n-j+1}}\right\}\langle\xi, \eta\rangle^{j} .
\end{aligned}
$$

By the recursive formulae (2.27), we have

$$
\left(\mathscr{S} C_{n+1}(x ; \eta)\right)(\xi)=\sum_{j=0}^{n}(-1)^{n-j} \frac{n !}{j !} j\left\{\left\langle\xi, \eta^{n-j+2}\right\rangle+\overline{\left.\eta^{n-j+2}\right\}}\langle\xi, \eta\rangle^{j-1}\right.
$$




$$
\begin{aligned}
& +\sum_{j=0}^{n}(-1)^{n-j} \frac{n !}{j !}\left\{\langle\xi, \eta\rangle^{n-j+1}+\overline{\eta^{n-j+1}}\right\}\langle\xi, \eta\rangle^{j}-\bar{\eta}\langle\xi, \eta\rangle^{n} \\
= & \langle\xi, \eta\rangle^{n+1} .
\end{aligned}
$$

Therefore $C_{n+1}(x ; \eta)$ belongs to $\mathscr{H}$ and $\left.\mathscr{S} C_{n+1}(x ; \eta)\right)(\xi)=\langle\xi, \eta\rangle^{n+1}$. Since $\mathscr{S}$ is an isomorphism,

$$
\left(C_{n}(x ; \eta), C_{m}(x ; \zeta)\right)_{\mathscr{x}(p)}=\delta_{n, m} n !(\eta, \zeta)_{p}^{n}
$$

holds for $\eta, \zeta \in \mathscr{E}$ by virtue of Proposition 3.4. Therefore the series

$$
f_{\eta}(x) \equiv \sum_{n=0}^{\infty} \frac{1}{n !} C_{n}(x ; \eta)
$$

converges strongly in $\mathscr{H}$ and the equality

$$
\left(\mathscr{S} f_{\eta}(\cdot)\right)(\xi)=\exp [\langle\xi, \eta\rangle]
$$

holds. Since

$$
\left\|C_{n}(x ; \eta)\right\|_{L^{1}\left(\xi^{*}, \mu\right)} \leq\left\|C_{n}(x ; \eta)\right\|_{L^{2}\left(\varepsilon^{*}, \mu\right)}=\left\|\langle\xi, \eta\rangle^{n}\right\|_{\mathscr{F}^{(0)}}=(n !)^{1 / 2}\|\eta\|_{0}^{n},
$$

the series (5.13) converges almost surely $\mu$. On the other hand, by the definition of $C_{n}(x ; \eta)$ 's,

$$
f_{\omega \eta}(x)=\sum_{n=0}^{\infty} \frac{\omega^{n}}{n !} C_{n}(x ; \eta)=\exp [\langle x, \log (1+\omega \eta)\rangle-\omega \bar{\eta}]
$$

holds, if $x \in E_{-r}$ and $|\omega| C_{r}\|\eta\|_{r}<1$. By the assumption,

$$
\exp \left[\langle x, \eta\rangle-\overline{\left(e^{\eta}-1\right)}\right]=\sum_{n=0}^{\infty} \frac{1}{n !} C_{n}\left(x ; e^{\eta}-1\right) \quad \text { a.s. }
$$

holds for $\eta \in \mathscr{E}$ with $C_{r}\left\{\exp \left[C_{r}\|\eta\|_{r}\right]-1\right\}<1$. Therefore, for $|\omega| \leq 1$,

$$
\int_{\delta^{*}} \exp \left[\omega\langle x, \eta\rangle-\int_{T}\left(e^{\omega \eta}-1\right) d \nu\right] d \mu=\left\langle\exp \left[\left\langle\xi, e^{\omega \eta}-1\right\rangle\right], 1\right\rangle=1
$$

is obtained. Hence we have

$$
\int_{\delta^{*}} \exp [\omega\langle x, \eta\rangle] d \mu=\exp \left[\int_{T}(\exp [\omega \eta(t)]-1) d \nu(t)\right]
$$

for $|\omega| \leq 1$. We can easily see that both sides are analytic in $\omega$. Thus we have the equality

$$
\int_{\dot{\sigma}^{*}} \exp [i\langle x, \eta\rangle] d \mu=\exp \left[\int_{T}(\exp [i \eta(t)]-1) d \nu(t)\right]
$$


which implies that $\mu$ is the measure of Poisson white noise.

THEOREM 5.5. If the basic triplet $\mathscr{E} \subset L^{2}(T, \nu) \subset \mathscr{E}^{*}$ satisfies the following property, then Theorem 5.4 is true without the assumption on the support of the measure $\mu$ : For any Borel set $A$ with $\nu(A)<\infty$, there exists an approximating sequence $\left\{\zeta_{k}\right\}$ included in $\mathscr{E}$ such that

$$
\int_{T}\left|\zeta_{k}(t)-\chi_{A}(t)\right| d \nu(t) \longrightarrow 0 \quad \text { as } k \rightarrow \infty
$$

and that $\zeta_{k}$ 's are uniformly bounded, i.e.

$$
\sup _{t \in T, k>0}\left|\zeta_{k}(t)\right|<\infty
$$

Proof. We can follow the counterpart of the stages before (5.14) in the proof of Theorem 5.4, in the same way. Let $\left\{\eta_{k}\right\}$ and $\left\{\zeta_{k}\right\}$ be uniform bounded sequences in $\mathscr{E}$ such that $\eta_{k} \rightarrow \eta, \zeta_{k} \rightarrow \zeta$ in $L^{1}(T, \nu)$. Since $\left\langle\xi, \eta_{k}\right\rangle^{n} \rightarrow\langle\xi, \eta\rangle^{n}$ and $\left\langle\xi, \zeta_{k}\right\rangle^{m} \rightarrow\langle\xi, \zeta\rangle^{m}$ in $\mathscr{F}^{(0)}$, the following limits exist in $L^{2}\left(\mathscr{E}^{*}, \mu\right)$;

$$
C_{n}(x ; \eta) \equiv \lim _{k \rightarrow \infty} C_{n}\left(x ; \eta_{k}\right) \quad \text { and } \quad C_{m}(x ; \eta)=\lim _{k \rightarrow \infty} C_{m}\left(x ; \zeta_{k}\right),
$$

even if $\eta, \zeta \notin \mathscr{E}$. By using the recursive formulae (2.27), we get

$$
C_{n+1}(x ; \eta)=\sum_{j=0}^{n}(-1)^{n-j} \frac{n !}{j !}\left\{C_{1}\left(x ; \eta^{n-j+1}\right)+\overline{\left.\eta^{n-j+1}\right\}} C_{j}(x ; \eta)-\bar{\eta} C_{n}(x ; \eta) .\right.
$$

Notice that

$$
C_{n}(x ; \eta) C_{m}(x ; \zeta)=\lim _{k \rightarrow \infty} C_{n}\left(x ; \eta_{k}\right) C_{m}\left(x ; \zeta_{k}\right) \quad \text { in } L^{1}(\mathscr{E} *, \mu) .
$$

Then by (5.12), we have that

$$
\left(\mathscr{S}\left(C_{1}\left(x ; \eta_{k}\right) C_{m}\left(x ; \zeta_{k}\right)\right)\right)(\xi)-\left\langle\xi, \eta_{k}\right\rangle\left\langle\xi, \zeta_{k}\right\rangle^{m}=m\left\langle 1+\xi, \eta_{k} \zeta_{k}\right\rangle\left\langle\xi, \zeta_{k}\right\rangle^{m-1} .
$$

Appealing to (2.27) by induction, we have that

$$
\left(\mathscr{S}\left(C_{n}\left(x ; \eta_{k}\right) C_{m}\left(x ; \zeta_{k}\right)\right)\right)(\xi)-\left\langle\xi, \eta_{k}\right\rangle^{n}\left\langle\xi, \zeta_{k}\right\rangle^{m}
$$

is a polynomial whose terms include either $\left\langle\xi, \eta_{k}^{i} \zeta_{k}^{j}\right\rangle$ or $\left\langle 1, \eta_{k}^{i} \zeta_{k}^{j}\right\rangle$ with $i, j \geq 1$. Now we assume that $\eta(t) \zeta(t)=0$ a.e. $t(\nu)$. Then letting $k \rightarrow \infty$, we get

$$
\left(\mathscr{S}\left(C_{n}(x ; \eta) C_{m}(x ; \zeta)\right)(\xi)=\langle\xi, \eta\rangle^{n}\langle\xi, \zeta\rangle^{m}\right.
$$

Therefore the equalities 


$$
\begin{aligned}
& \sum_{m=0}^{n} \frac{n ! \omega^{n-m} \lambda^{m}}{(n-m) ! m !} C_{n-m}(x ; \eta) C_{m}(\chi ; \zeta) \\
& \left.\quad=\mathscr{S}^{-1}\left(\sum_{m=0}^{n} \frac{n ! \omega^{n-m} \lambda^{m}}{(n-m) ! m !}\langle\xi, \eta\rangle^{n}\langle\xi, \zeta\rangle^{m}\right\rangle\right) \\
& \quad=\mathscr{S}^{-1}\left(\langle\xi, \omega \eta+\lambda \zeta\rangle^{n}\right)=C_{n}(x ;(\omega \eta+\lambda \zeta))
\end{aligned}
$$

hold. Let $A_{j}, 1 \leq j \leq L$, be disjoint Borel sets with $\nu\left(A_{j}\right)<\infty$, and let $\omega_{j}, 1 \leq j \leq L$, be real numbers. By the property (ii), we can apply (5.17) to $\sum_{j} \chi_{A_{j}}(t)$, and we get

$$
C_{n}\left(x ; \sum_{j=1}^{L} \omega_{j} \chi_{A_{j}}\right)=\sum_{n_{1}+\cdots+n_{L}=n} \frac{n ! \omega_{1}^{n_{1}} \cdots \omega_{L}^{n_{L}}}{n_{1} ! \cdots n_{L} !} C_{n_{1}}\left(x ; \chi_{A_{1}}\right) \cdots C_{n_{L}}\left(x ; \chi_{A_{L}}\right) .
$$

On the other hand, we have

$$
\begin{aligned}
C_{n+1}\left(x ; \chi_{A}\right)= & \sum_{j=0}^{n}(-1)^{n-j} \frac{n !}{j !}\left\{C_{1}\left(x ; \chi_{A}\right)+\nu(A)\right\} C_{j}\left(x ; \chi_{A}\right) \\
& -\nu(A) C_{n}\left(x ; \chi_{A}\right)
\end{aligned}
$$

by (5.15). Define the usual Charlier polynomials $C_{n}(u ; \lambda)$ with parameter $\lambda$ by the generating function

$$
\exp [u \log (1+\omega)-\omega \lambda]=\sum_{n=0}^{\infty} \frac{\omega^{n}}{n !} C_{n}(u ; \lambda) .
$$

Then $C_{n}(u ; \lambda)$ 's satisfy the recursive formulae

$$
\left\{\begin{array}{l}
C_{n+1}(u ; \lambda)=\sum_{j=0}^{n}(-1)^{n-j} \frac{n !}{j !} u C_{n}(u ; \lambda)-\lambda C_{n}(u ; \lambda), \\
C_{0}(u ; \lambda)=1 \text { and } C_{1}(u ; \lambda)=u-\lambda .
\end{array}\right.
$$

Put $P(A)=C_{1}\left(x ; \chi_{A}\right)+\nu(A)$, then we have by (5.19)

$$
C_{n}\left(x ; \chi_{A}\right)=C_{n}(P(A) ; \nu(A))
$$

and more generally by (5.18)

$$
\begin{aligned}
\sum_{n=0}^{\infty} \frac{1}{n !} & C_{n}\left(x ; \sum_{k=1}^{L} \omega_{k} \chi_{A k}\right) \\
& =\sum_{n_{1}+\cdots+n_{L}=n} \frac{\omega_{1}^{n_{1}} \cdots \omega_{L}^{n_{L}}}{n_{1} ! \cdots n_{L} !} C_{n_{1}}\left(P\left(A_{1}\right) ; \nu\left(A_{1}\right)\right) \cdots C_{n_{\mathbf{L}}}\left(P\left(A_{L}\right) ; \nu\left(A_{L}\right)\right) \\
& =\exp \left[\sum_{k=1}^{L} P\left(A_{k}\right) \log \left(1+\omega_{k}\right)-\sum_{k=1}^{L} \omega_{k} \nu\left(A_{k}\right)\right] .
\end{aligned}
$$

Hence 


$$
\begin{gathered}
\int_{\mathfrak{c}^{*}} \exp \left[\sum_{k=1}^{L} P\left(A_{k}\right) \log \left(1+\omega_{k}\right)-\sum_{k=1}^{L} \omega_{K} \nu\left(A_{k}\right)\right] d \mu \\
=\left(\mathscr{S}\left(\sum_{n=0}^{\infty} \frac{1}{n !} C_{n}\left(x ; \sum_{k=1}^{L} \omega_{k} \chi_{A_{k}}\right)\right)(\xi), 1\right)_{\mathscr{F}^{(0)}} \\
=\left(\exp \left[\left\langle\xi, \sum_{k=1}^{L} \omega_{k} \chi_{A_{k}}\right\rangle\right], 1\right)_{\mathscr{F}^{(0)}}=1
\end{gathered}
$$

applying (5.16) with $\xi=0, m=1$. Therefore for any reals $\omega_{k}, 1 \leq k \leq L$, we have

$$
\int_{\varepsilon^{*}} \exp \left[\sum_{k=1}^{L} \omega_{k} P\left(A_{k}\right)\right] d \mu=\exp \left[\sum_{k=1}^{L}\left(\exp \left[\omega_{k}\right]-1\right) \nu\left(A_{k}\right)\right] .
$$

This implies that $\mu$ is the measure of Poisson white noise.

\section{§ 6. Wick's normal ordering}

By (2.8), the canonical commutation relations

$$
\left\{\begin{array}{l}
{\left[\partial_{t}, \partial_{s}^{*}\right]=\delta_{s}(t),} \\
{\left[\partial_{t}, \partial_{s}\right]=0 \text { and }\left[\partial_{t}^{*}, \partial_{s}^{*}\right]=0,}
\end{array}\right.
$$

are given. Hence Wick's normal ordering is applicable to our calculus as pointed out in [11] for Gaussian case.

Let $\gamma_{t_{j}}=\partial_{t_{j}}$ or $\partial_{t_{j}}^{*}, j=1,2, \cdots, n$, and put $J \equiv\left\{j ; \gamma_{t_{j}}=\partial_{t_{j}}\right\}, J^{*}=$ $\left\{j ; \gamma_{t_{j}}=\partial_{t_{j}}^{*}\right.$. Then Wick's normal ordering is defined by

$$
: \gamma_{t_{1}} \cdots \gamma_{t_{n}}:=\prod_{j \in J^{*}} \partial_{t_{j}}^{*} \prod_{i \in J} \partial_{t_{i}} \text {. }
$$

Then $: \gamma_{t_{1}} \ldots \gamma_{t_{n}}:$ is a continuous operator from $\mathscr{H}_{X}$ into $\mathscr{H}_{X}^{*}$ (or more precisely from $\mathscr{H}_{X}^{(1)}$ into $\mathscr{H}_{X}^{(-1)}$, though $\gamma_{t_{1}} \cdots \gamma_{t_{n}}$ has meaning only as an operator valued generalized function. For any formal power series $\mathscr{P}\left(\partial_{t_{1}}, \cdots, \partial_{t_{n}}, \partial_{s_{1}}^{*}, \cdots, \partial_{s_{m}}^{*}\right)$ of free algebra generated by $\partial_{t_{1}}, \cdots, \partial_{t_{n}}, \partial_{s_{1}}^{*}, \cdots, \partial_{s_{m}}^{*}$,

$$
: \mathscr{P}\left(\partial_{t_{1}}, \cdots, \partial_{t_{n}}, \partial_{s_{1}}^{*}, \cdots, \partial_{s_{m}}^{*}\right):
$$

is defined by operating : : to each term.

EXAMPLE 6.1. As an operator valued generalized function,

$$
\partial_{t} \partial_{s}^{*} \partial_{r}^{*}=\delta_{s}(t) \partial_{r}^{*}+\delta_{r}(t) \partial_{s}^{*}+\partial_{s}^{*} \partial_{r}^{*} \partial_{t}
$$

holds by (6.1), but

$$
\partial_{s}^{*} \partial_{r}^{*} \partial_{t}=: \partial_{t} \partial_{s}^{*} \partial_{r}^{*}: \neq: \delta_{s}(t) \partial_{r}^{*}+\delta_{r}(t) \partial_{r}^{*}+\partial_{s}^{*} \partial_{r}^{*} \partial_{\iota}: .
$$


To see the action of $\partial_{t_{1}} \cdots \partial_{t_{n}}$, we want to rewrite it similarly to the example. Here we introduce a notation: let $\left[J, J^{*}\right]$ be the collection of all sets of pairs $\left[K, K^{*}\right]=\left\{\left(k_{j}, k_{j}^{*}\right)\right\}_{j=1}^{m}$ such that $k_{j}<k_{j}^{*}$ and that $K=\left\{k_{1}, \cdots, k_{m}\right\}$ are distinct elements of $J$ and $K^{*}=\left\{k_{1}^{*}, \cdots, k_{m}^{*}\right\}$ are distinct elements of $J^{*}$. Then âsplying (6.1), we have

$$
\gamma_{t_{1}} \cdots \gamma_{t_{n}}=\sum_{\left[K, K^{*}\right] \subset\left[J, J^{*}\right]} \prod_{j=1}^{m} \delta_{t_{k_{j}^{*}}}\left(t_{k_{j}}\right) \prod_{i \in J^{*} \backslash K^{*}} \partial_{t_{i}}^{*} \prod_{h \in J \backslash K} \partial_{t_{h}}
$$

Thus for $f_{n}\left(t_{1}, \cdots, t_{n}\right) \in \mathscr{E}^{\hat{\otimes}} n$, a continuous operator

$$
\int_{T^{n}} f_{n}(\boldsymbol{t}) d \nu^{n}(\boldsymbol{t}) r_{t_{1}} \cdots r_{t_{n}}
$$

on $\mathscr{H}_{X}$ is well defined by Lemma 4.6 and by Proposition 4.5 for $X=P$ and $G$.

Let us observe $x^{P}\left(t_{1}\right) \cdots x^{P}\left(t_{n}\right)$. on $\mathscr{H}_{P}$, here we denote $x^{P}(t)=$ $\left(\partial_{t}^{*}+1\right)\left(\partial_{t}+1\right)$. By the definition of : :, we have

$$
\begin{aligned}
& \left.: x^{P}\left(t_{1}\right) \cdots x^{P}\left(t_{n}\right) \cdot:=\sum_{J, J^{*} \subset\{1} \cdots, n\right\} \\
& :\left(\prod_{k \in J^{*}} \partial_{t_{k}}^{*} \prod_{j \in J} \partial_{t_{j}},\right. \\
& \quad=x_{J^{*}+K+J=\{1, \cdots, n\}} \sum_{i \in J^{*}} \partial_{t_{i}}^{*} \prod_{k \in K} \partial_{t_{k}}^{*} \prod_{k \in K} \partial_{t_{k}} \prod_{j \in J} \partial_{t_{j}} .
\end{aligned}
$$

Therefore, for $f_{n} \in \mathscr{E}^{\hat{\otimes} n}$,

$$
\begin{gathered}
\int_{T^{n}} d \nu^{n}(\boldsymbol{t}) f_{n}(\boldsymbol{t}):\left(x^{P}\left(t_{1}\right) \cdot-1\right) \cdots\left(x^{P}\left(t_{n}\right) \cdot-1\right): \\
=\sum_{J^{*+K}+J=\{1, \cdots, n\}} A_{\left|J^{*},\right| K|,| J \mid}\left(f_{n}\right) \\
=\sum_{j^{*+k+j=n}} \frac{n !}{j^{*} ! k ! j !} A_{j^{*}, k, j}\left(f_{n}\right) .
\end{gathered}
$$

In particular, we have

$$
\left\{\begin{array}{l}
:\left(x^{P}\left(t_{1}\right) \cdot-1\right) \cdots\left(x^{P}\left(t_{n}\right) \cdot-1\right): 1=\partial_{t_{1}}^{*} \cdots \partial_{t_{n}}^{*} 1 \\
\int_{T^{n}} d \nu^{n}(\boldsymbol{t}) f_{n}(\boldsymbol{t}):\left(x^{P}\left(t_{1}\right) \cdot-1\right) \cdots\left(x^{P}\left(t_{n}\right) \cdot-1\right): 1=A^{*}\left(f_{n}\right) 1 .
\end{array}\right.
$$

TheOREM 6.2. For $f_{n} \in \mathscr{E}^{\hat{\otimes} n}$, the following holds in $\mathscr{H}_{P}^{*}$ :

$$
I_{n}^{P}\left(f_{n}\right)=A^{*}\left(f_{n}\right) 1=\int_{T^{n}} d \nu^{n}(\boldsymbol{t}) f_{n}(\boldsymbol{t}):\left(x^{P}\left(t_{1}\right) \cdot-1\right) \cdots\left(x^{P}\left(t_{n}\right) \cdot-1\right): 1 .
$$

Moreover for $\varphi \in \mathscr{H}_{P}$, the multiplication $I_{n}^{P}\left(f_{n}\right) \varphi$ belongs to $\mathscr{H}_{P}$ and 


$$
\begin{aligned}
I_{n}^{P}\left(f_{n}\right) \varphi & =\int_{T^{n}} d \nu^{n}(\boldsymbol{t}) f_{n}(\boldsymbol{t}):\left(x^{P}\left(t_{1}\right) \cdot-1\right) \cdots\left(x^{P}\left(t_{n}\right) \cdot-1\right): \varphi \\
& =\left(A^{*}\left(f_{n}\right) 1\right) \varphi \neq A^{*}\left(f_{n}\right) \varphi .
\end{aligned}
$$

Proof. The first assertion follows from the above discussion and from Theorem 4.7. Since (5.7) holds for $\eta$ and $\zeta$ in $\mathscr{E}$, we have

$$
\left(\mathscr{S}_{P}\left(C_{n}(x ; \eta) f^{P}(\zeta ; x)\right)\right)(\xi)=(\langle\xi, \eta \zeta+\eta\rangle+\langle\eta, \zeta\rangle)^{n} \exp [\langle\xi, \zeta\rangle]
$$

by (2.21) and (2.23). On the other hand,

$$
\begin{aligned}
\mathscr{S}_{P}\left(\int_{T^{n}}\right. & \left.d \nu^{n}(\boldsymbol{t}) \eta\left(t_{1}\right) \cdots \eta\left(t_{n}\right):\left(x^{P}\left(t_{1}\right) \cdot-1\right) \cdots\left(x^{P}\left(t_{n}\right) \cdot-1\right): f^{P}(\zeta ; x)\right)(\xi) \\
& =\mathscr{S}_{P}\left(\sum_{j^{*}+k+j=n} \frac{n !}{j^{*} ! k ! j !} A^{*}(\eta)^{\jmath^{*}} A_{0, k, 0}\left(\eta^{\hat{\otimes} k}\right) A(\eta)^{j} f^{P}(\zeta ; x)\right)(\xi) \\
& =\sum_{j^{*}+k+j=n} \frac{n !}{j^{*} ! k ! j !}\langle\xi, \eta\rangle^{j^{*}}\langle\xi, \eta \zeta\rangle^{k}\langle\xi, \zeta\rangle^{j} \exp [\langle\xi, \zeta\rangle] \\
& =(\langle\xi, \eta\rangle+\langle\xi, \eta \zeta\rangle+\langle\xi, \zeta\rangle)^{n} \exp [\langle\xi, \zeta\rangle]
\end{aligned}
$$

holds by (6.6), (4.5) and Proposition 4.4. Therefore the assertion in the theorem holds for $f_{n}=\eta^{\hat{\otimes}}$ on $\dot{\mathscr{H}}_{P}$, because $C_{n}(x ; \eta)=A^{*}\left(\eta^{\hat{\otimes} n}\right) 1$. By Proposition 4.5, we can show that the assertion is true on $\mathscr{H}_{p}$, similarly to the proof of Theorem 5.1. Therefore it is true for $f_{n}=\sum c_{j} \eta_{j}^{\hat{\otimes} n}, \eta_{j} \in \mathscr{E}$. Applying Proposition 4.5 again, we complete the proof.

The corresponding theorem for Gaussian case is given in [12].

THEOREM 6.3. (i) For $f_{n} \in \mathscr{E}^{\otimes} \mathrm{n}$,

$$
I_{n}^{G}\left(f_{n}\right)=A^{*}\left(f_{n}\right) 1=\int_{T^{n}} d \nu^{n}(\boldsymbol{t}) f_{n}(\boldsymbol{t}): x^{G}\left(t_{1}\right) \cdots x^{G}\left(t_{n}\right) \cdot: 1
$$

holds in $\mathscr{H}_{G}$. Moreover, for $\varphi \in \mathscr{H}_{G}, I^{G}\left(f_{n}\right) \varphi$ belongs to $\mathscr{H}_{G}$ and

$$
\begin{aligned}
I_{n}^{G}\left(f_{n}\right) \varphi & =\int_{T^{n}} d \nu^{n}(t) f_{n}(\boldsymbol{t}): x^{G}\left(t_{1}\right) \cdots x^{G}\left(t_{n}\right) \cdot: \varphi \\
& =\left(A^{*}\left(f_{n}\right) 1\right) \varphi \neq A^{*}\left(f_{n}\right) \varphi .
\end{aligned}
$$

(ii) The multiplication $(\psi, \varphi) \rightarrow \psi \varphi$ is continuous in $\mathscr{H}_{G}$, actually,

$$
\|\psi \varphi\|_{\mathscr{x}_{G}^{(p)}} \leq 5\|\psi\|_{\mathscr{X}_{G}^{(p+q)}}\|\varphi\|_{\mathscr{X}_{G}^{(p+q)}}
$$

holds, if $\rho^{q}\left(4+\|\delta\|^{2}\right)<1$.

Let us define the vacuum expectation of an operator $A$ by

$$
\langle A\rangle=\langle A 1,1\rangle \text {. }
$$


Then $\left\langle x^{G}(t) \cdot\right\rangle=0$ and $\left\langle x^{P}(t) \cdot\right\rangle=1$ hold. Hence the following common expression of the multiple Wiener integrals holds:

TheOREM 6.4. For $f_{n} \in \mathscr{E}^{* \hat{\otimes}}, \varphi \in \mathscr{H}_{X}, X=P, G$, it holds that

$$
I_{n}^{X}\left(f_{n}\right)=\int_{\boldsymbol{R}^{n}} d \boldsymbol{t} f_{n}(\boldsymbol{t}):\left(x^{X}\left(t_{1}\right) \cdot-\left\langle x^{X}\left(t_{1}\right) \cdot\right\rangle\right) \cdots\left(x^{X}\left(t_{n}\right) \cdot-\left\langle x^{X}\left(t_{n}\right) \cdot\right\rangle\right): \varphi .
$$

Unfortunately, the assertion (ii) of Theorem 6.3 is not true for the Poisson case. For example, take $\varphi=f^{P}(\eta ; x)$ and $\psi=f^{P}(\zeta ; x)$. Then the norms of $\psi \varphi=f^{P}(\zeta \eta+\zeta+\eta ; x) \exp [\langle\zeta, \eta\rangle]$ is calculated as

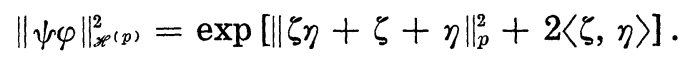

Actually, it is not bounded by constant times of $\|\psi\|_{\mathscr{P}^{(p+q)}}^{2}\|\varphi\|_{\mathscr{P}^{(p+q)}}^{2}=$ $\exp \left[\|\zeta\|_{p+q}^{2}+\|\eta\|_{p+q}^{2}\right]$.

Proposition 6.5. (i) If $\psi=\sum_{n=0}^{\infty} I_{n}^{P}\left(f_{n}\right) \in \mathscr{H}_{P}$ satisfies

$$
\sum_{n=0}^{\infty} n ! a^{2 n}\left\|I_{n}^{P}\left(f_{n}\right)\right\|_{\mathscr{C}_{P}^{(p)}}^{2}<\infty
$$

with some $a=a(p)>0$ for any $p$, then the mapping

$$
\psi \cdot: \varphi \longrightarrow \psi \varphi
$$

is continuous on $\mathscr{H}_{P}$.

(ii) For $\eta \in \mathscr{E}, \varphi \rightarrow f^{P}(\eta ; x) \varphi$ is a continuous mapping on $\mathscr{H}_{P}$.

Proof. By (6.6), Theorem 6.2 and Proposition 4.5 (ii), the assertion (i) is easily seen. Since

$$
\begin{aligned}
& f^{P}(\eta ; x)=\sum_{n=0}^{\infty} \frac{1}{n !} I_{n}\left(\eta^{\hat{\otimes} n}\right) \text { and } \\
& \sum_{n=0}^{\infty} \frac{n !}{(n !)^{2}}\left(1+\|\eta\|_{p}^{2}\right)^{-n}\left\|I_{n}\left(\eta^{\hat{\otimes} n}\right)\right\|_{\mathscr{X}^{(}(p)}^{2}=\sum_{n=0}^{\infty}\left(1+\|\eta\|_{p}^{2}\right)^{-n}\|\eta\|_{p}^{2 n}<\infty,
\end{aligned}
$$

(ii) is obvious by (i).

Let $f_{n}$ be in $\mathscr{E}^{\otimes} \otimes$, then $\left\langle x^{\hat{\otimes} n}, f_{n}\right\rangle$ is a continuous functional defined on $\mathscr{E}^{*}$. Can we show that $\left\langle x^{\hat{\otimes} n}, f_{n}\right\rangle$ belongs to $\mathscr{H}_{P}$ (resp. to $\mathscr{H}_{G}$ )? Firstly, we see that for $\eta_{1}, \cdots, \eta_{n} \in \mathscr{E}$,

$$
\begin{aligned}
\left\langle x^{\hat{\otimes} n}, \eta_{1} \hat{\otimes} \cdots \hat{\otimes} \eta_{n}\right\rangle & =\left\langle x, \eta_{1}\right\rangle \cdots\left\langle x, \eta_{n}\right\rangle \cdot 1 \\
& =\left(I^{P}\left(\eta_{1}\right)+\bar{\eta}_{1}\right) \cdots\left(I^{P}\left(\eta_{n}\right)+\bar{\eta}_{n}\right)
\end{aligned}
$$

belongs to $\mathscr{H}_{P}$ by Theorem 6.2. Further we can prove 


$$
\left\langle x^{\hat{\otimes} n}, \eta_{1} \hat{\otimes} \cdots \hat{\otimes} \eta_{n}\right\rangle=\int_{T^{n}} d \nu^{n}(t) \eta_{1}\left(t_{1}\right) \eta_{1} \cdots \eta_{n}\left(t_{n}\right) x^{P}\left(t_{1}\right) \cdots x^{P}\left(t_{n}\right) \cdot 1 .
$$

For $f_{n} \in \mathscr{E}^{\mathscr{E}^{\hat{\otimes}} n}$, we have an expression

$$
\int_{T^{n}} d \nu^{n}(\boldsymbol{t}) f_{n}(\boldsymbol{t}) x^{P}\left(t_{1}\right) \cdots x^{P}\left(t_{n}\right) \cdot \varphi=\sum_{j^{*}+k \leq n, j+k \leq n} A_{j^{*}, k, j}\left(f_{n ; j^{*}, k, j}\right) \varphi,
$$

by using (6.3), with the continuous linear mappings defined by

$$
\begin{aligned}
& f_{n} \longrightarrow f_{n ; j^{*}, k, j} \in \mathscr{E}^{\hat{\otimes}\left(j^{*}+k+j\right)}, \\
& f_{n ; j^{*}, k, j}\left(t_{1}, \cdots, t_{j^{*}+k+j}\right) \\
& \quad=\sum_{n \geq m \geq j^{*}+k+j} \frac{m !(m-k) !}{k ! j^{* ! j !}\left(m-k-j^{*}\right) !(m-k-j) !} \int f_{n ; m}(\boldsymbol{t}, s) d \nu^{m-j^{*-k-j}}(s), \\
& \quad f_{n ; m}\left(t_{1}, t_{2}, \cdots, t_{m}\right) \\
& \quad=\sum_{\substack{r_{1}+\cdots++r_{s}=m \\
k_{1} r_{1}+\cdots+k_{s} r_{s}=n}} \frac{n !}{m !\left(k_{1} !\right)^{r_{1}} \cdots\left(k_{s} !\right)^{r}{ }_{s}} \prod_{i=1}^{r_{1}} \delta_{t_{i}}^{\hat{\otimes} k_{1}} * \cdots\left(\prod_{i=m-r_{s}+1}^{m} \delta_{t_{i}}^{\hat{\otimes}_{k} k_{s}} *\right) f_{n},
\end{aligned}
$$

Applying Proposition 4.5, we can see:

Proposition 6.6. For $f_{n} \in \mathscr{E}^{\stackrel{\otimes}{\otimes} n}$, it holds that

$$
\left\langle x^{\hat{\otimes} n}, f_{n}\right\rangle \cdot=\int_{T^{n}} d \nu^{n}(t) f_{n}(t) x^{X}\left(t_{1}\right) \cdots x^{X}\left(t_{n}\right) .
$$

on $\mathscr{H}_{X}$ for $X=P$ and also $G$.

Proof. In the case of Poisson, the assertion is clear by the above discussion. We can check for the Gaussian case similarly.

Remark 6.7. For the Gaussian case, we have

$$
\left\langle x^{\hat{\otimes} n}, f_{n}\right\rangle=\sum_{k=0}^{[n / 2]} \frac{n ! 2^{-k}}{(n-2 k) ! k !} I_{n-2 k}\left(f_{n \mid n-2 k}\right)
$$

with

$$
f_{n \backslash n-2 k}\left(t_{1}, \cdots, t_{n-2 k}\right) \equiv \int_{T 2 k} f_{n}\left(t_{1}, \cdots, t_{n-2 k}, s_{1}, s_{1}, \cdots, s_{k}, s_{k}\right) d \nu^{k} .
$$

Remark 6.8. For $f_{n} \in \mathscr{S}(\boldsymbol{R})^{\hat{\otimes} n}$, we have that

$$
\int_{\boldsymbol{R}^{n}} d \boldsymbol{t} f_{n}(\boldsymbol{t}): x^{P}\left(t_{1}\right) \cdots x^{P}\left(t_{n}\right): 1=\sum_{k=0}^{n}\left(\begin{array}{l}
n \\
k
\end{array}\right) I_{k}^{P}\left(f_{k}\right)
$$

where $f_{k}$ is defined by

$$
f_{k}\left(t_{1}, \cdots, t_{k}\right) \equiv \int_{\boldsymbol{R}^{n-k}} f_{n}\left(t_{1}, \cdots, t_{k}, u_{1}, \cdots, u_{n-k}\right) d u .
$$




\section{§7. Remarks}

In the Gaussian case, the transformation $\mathscr{S}_{G}$ has a beautiful expression

$$
\left(\mathscr{S}_{G} \varphi\right)(\xi)=\int_{\epsilon^{*}} \varphi(x+\xi) d \mu_{G}(x)
$$

which clarifies the reason why $\partial_{t}$ gives a derivation (cf. [11]). It should be an essential point that the generator of the shift $x+\xi$ is the derivative. Are there any similar explanations of the transformation $\mathscr{S}_{P}$ ? We now discuss on the problem. Let us consider a Poisson random variable $X$ with mean $\lambda$. In the analysis of $\varphi=\varphi(X)$, shift $\varphi(x+\xi)$ does not have meaning, because $X$ takes its values only on $Z_{+}=\{0,1,2, \cdots\}$ with probabilities

$$
\mu_{P}(X=x)=\frac{\lambda^{X}}{x !} \exp [-\lambda]
$$

However, the unit shift $(\sigma \varphi)(x) \equiv \varphi(x+1)$ and difference

$$
(\Delta \varphi)(x)=(\sigma-1) \varphi(x)=\varphi(x+1)-\varphi(x)
$$

should be useful. Let $\tau_{\xi}$ be the semi-group generated by the difference $\Delta$;

$$
\begin{aligned}
\left(\tau_{\xi} \varphi\right)(x) & \equiv \sum_{n=0}^{\infty} \frac{(\xi \Delta)^{n}}{n !} \varphi(x) \\
& =\exp [\xi(\sigma-I)] \varphi(x)=(\exp [\xi \sigma] \varphi)(x) \exp [-\xi] \\
& =\sum_{k=0}^{\infty} \frac{\xi^{k}}{k !} \varphi(x+k) \exp [-\xi]
\end{aligned}
$$

Then, the expectation of $\varphi$ is given by

$$
\int \varphi(X) d \mu_{P}=\left(\tau_{2} \varphi\right)(0) .
$$

Now we define a transformation $\mathscr{S}$ by

$$
(\mathscr{S} \varphi)(\xi) \equiv \int\left(\tau_{\lambda \xi} \varphi\right)(X) d \mu_{P} .
$$

Then, by (7.3), (7.4) and by the group property of $\left\{\tau_{\varepsilon}\right\}$, we have

$$
\begin{aligned}
(\mathscr{S} \varphi)(\xi) & =\left(\left(\tau_{\lambda} \tau_{\lambda \xi}\right) \varphi\right)(0)=\left(\tau_{\lambda(1+\xi)} \varphi\right)(\theta) \\
& =\sum_{x=0}^{\infty} \frac{(\lambda(1+\xi))^{x}}{x !} \varphi(x) \exp [-\lambda-\lambda \xi]
\end{aligned}
$$




$$
\begin{aligned}
& =\int(1+\xi)^{X} \varphi(X) \exp [-\lambda \xi] d \mu_{P} \\
& =\int \varphi(X) \exp [X \log (1+\xi)-\lambda \xi] d \mu_{P} .
\end{aligned}
$$

The last expression corresponds to the definition of $\mathscr{S}_{P}$. Put $U(\xi)=\left(\mathscr{S}_{\varphi}\right)(\xi)$, then

$$
U^{(1)}(\xi)=\mathscr{S}((\lambda \Delta) \varphi)(\xi)
$$

holds. Therefore

$$
\partial \varphi \equiv \mathscr{S}^{-1} \frac{d}{d \xi} \mathscr{S}_{\varphi}=\lambda \Delta \varphi
$$

Since

$$
\begin{aligned}
\int(\sigma \varphi)(X) \psi(X) d \mu_{P} & =\sum_{k=0}^{\infty} \frac{\lambda^{k}}{k !} \varphi(k+1) \psi(k) \exp [-\lambda] \\
& =\sum_{k=1}^{\infty} \frac{\lambda^{k}}{k !} \varphi(k) \frac{k}{\lambda} \psi(k-1) \exp [-\lambda],
\end{aligned}
$$

the dual of unit shift is given by

$$
\sigma^{*} \varphi(x)= \begin{cases}\frac{x}{\lambda} \varphi(x-1) & x \geq 1 \\ 0 & x=0 .\end{cases}
$$

Therefore we have

$$
\partial^{*}=(\lambda \Delta)^{*} \varphi=\lambda\left(\sigma^{*}-I\right) \varphi= \begin{cases}x \varphi(x-1)-\lambda \varphi(x) & x \geq 1 \\ -\lambda \varphi(0) & x=0 .\end{cases}
$$

For example, for Charlier's polynomials given by (5.20), we have

$$
\begin{aligned}
& \partial^{*} 1=x-\lambda, \\
& \partial^{*} \partial^{*} 1=x^{2}-(2 \lambda+1) x+\lambda^{2}, \\
& \vdots \\
& \left(\partial^{*}\right)^{n} 1=C_{n}(x ; \lambda) .
\end{aligned}
$$

Our transformation $\mathscr{S}_{P}$ can be considered as a continuous version of $\mathscr{S}$. Then $\hat{e}_{t}$ should be the difference operator;

$$
\partial_{t} \varphi(x)=\varphi\left(x+\delta_{t}\right)-\varphi(x)
$$

on $\mathscr{H}_{P}$. We have not yet known whether $\varphi$ in $\mathscr{H}_{P}$ is a continuous functional on $\mathscr{E}^{*}$ or not (for $\mathscr{H}_{G}$, a positive answer has been given [13] 
Remark 12.6). However, for densely many continuous functionals in $\mathscr{H}_{P}$, (7.10) is true.

Example 7.1. For $\eta$ in $\mathscr{E}$, the equalities

$$
\begin{aligned}
\partial_{t} \exp [\langle x, \eta\rangle] & =(\exp [\eta(t)]-1) \exp [\langle x, \eta\rangle] \\
& =\exp \left[\left\langle x+\delta_{t}, \eta\right\rangle\right]-\exp [\langle x, \eta\rangle],
\end{aligned}
$$

hold in $\mathscr{H}_{P}$. For $\eta \in \mathscr{E}$, the equality

$$
\partial_{t} f^{P}(\eta ; x)=\eta(t) f^{P}(\eta ; x)
$$

holds. If $\eta \in \mathscr{E}$, then we have

$$
\eta(t) f^{P}(\eta ; x)=f^{P}\left(\eta ; x+\delta_{t}\right)-f^{P}(\eta ; x) .
$$

Proof. Since $\langle x, \eta\rangle=I^{P}(\eta ; x)+\bar{\eta}$ holds for $\eta \in \mathscr{E}$, we get

$$
\left(\mathscr{S}_{P} \exp [\langle x, \eta\rangle]\right)(\xi)=\exp \left[\int_{T}(\exp [\eta(t)]-1)(\xi(t)+1) d \nu(t)\right]
$$

by (2.22). Therefore

$$
\frac{\delta}{\delta \xi(t)}\left(\mathscr{S}_{P} \exp [\langle x, \eta\rangle]\right)(\xi)=\{\exp [\eta(t)]-1\}\left(\mathscr{S}_{P} \exp [\langle x, \eta\rangle]\right)(\xi) .
$$

Thus we have the first assertion. By (2.21), it holds that

$$
\left(\mathscr{S}_{P} f^{P}(\eta ; x)\right)(\xi)=\exp [\langle\xi, \eta\rangle]
$$

and hence that

$$
\frac{\delta}{\delta \xi(t)}\left(\mathscr{S}_{P} f^{P}(\eta ; x)\right)(\xi)=\eta(t) \exp [\langle\xi, \eta\rangle]
$$

These imply the second assertion. If $\eta \in \mathscr{E}$, then we have that

$$
\begin{aligned}
f^{P}\left(\eta ; x+\delta_{t}\right) & =\exp \left[\left\langle x+\delta_{t}, \log (1+\eta)\right\rangle-\bar{\eta}\right] \\
& =\exp [\log (1+\eta(t))] f^{P}(\eta ; x)=(1+\eta(t)) f^{P}(\eta ; x)
\end{aligned}
$$

by (2.19).

EXAmple 7.2. For $\eta \in \mathscr{E}, f_{n} \in \mathscr{E}^{\hat{\otimes} n}$, we have

$$
\begin{gathered}
\partial_{t}\langle x, \eta\rangle^{n}=(\langle x, \eta\rangle+\eta(t))^{n}-\langle x, \eta\rangle^{n}, \\
\partial_{t} C_{n}(x ; \eta)=n \eta(t) C_{n-1}(x ; \eta)=C_{n}\left(x+\delta_{t} ; \eta\right)-C_{n}(x ; \eta), \\
\partial_{t}\left\langle x^{\hat{\otimes} n}, f_{n}\right\rangle=\left\langle\left(x+\delta_{t}\right)^{\hat{\otimes} n}, f_{n}\right\rangle-\left\langle x^{\hat{\otimes} n}, f_{n}\right\rangle
\end{gathered}
$$

in $\mathscr{H}_{\boldsymbol{p}}$. 
Proof. By the recursion formula (2.27), we see that $C_{n}(x ; \eta)$ is a continuous functional of $x$ in $\mathscr{E} *$. Suppose that

$$
C_{j}\left(x+\delta_{t} ; \eta\right)-C_{j}(x ; \eta)=j \eta(t) C_{j-1}(x ; \eta), \quad 1 \leq j \leq n .
$$

Then, by (2.27) again,

$$
\begin{aligned}
C_{n+1}\left(x+\delta_{t} ; \eta\right)-C_{n+1}(x ; \eta) \\
=\sum_{j=0}^{n}(-1)^{n-j} \frac{n !}{j !} \eta(t)^{n-j+1}\left\{C_{j}(x ; \eta)+j \eta(t) C_{j-1}(x ; \eta)\right\} \\
\quad+\sum_{j=0}^{n}(-1)^{n-j} \frac{n !}{j !}\left\langle x, \eta^{n-j+1}\right\rangle j \eta(t) C_{j-1}(x ; \eta)-n \bar{\eta} \eta(t) C_{n-1}(x ; \eta) \\
=\eta(t)\left\{C_{n}(x ; \eta)+n \sum_{j=0}^{n-1}(-1)^{n-1-j} \frac{(n-1) !}{j !}\left\langle x, \eta^{n-j}\right\rangle C_{j}(x ; \eta)-n \bar{\eta} C_{n-1}(x ; \eta)\right\} \\
=\eta(t)(n+1) C_{n}(x ; \eta) .
\end{aligned}
$$

On the other hand, by (2.24) we have that

$$
\begin{aligned}
\frac{\delta}{\delta \xi(t)}\left(\mathscr{S}_{P} C_{n+1}(x ; \eta)\right)(\xi) & =(n+1) \eta(t)\langle\xi, \eta\rangle^{n+1} \\
& =(n+1) \eta(t)\left(\mathscr{S}_{P} C_{n}(x ; \eta)\right)(\xi)
\end{aligned}
$$

Thus we have the second assertion. Applying (2.27) we can see that $\langle x, \eta\rangle^{n}$ can be represented by a linear combination of $\left\{C_{j}\left(x ; \eta^{k}\right) ; 0 \leq j, k \leq n\right\}$ (see [9]). Therefore the first assertion is true. The last one is proved by applying (6.9).

\section{REFERENCES}

[1] Aronszajn, N., Theory of reproducing kernel, Trans. Amer. Math. Soc., 68 (1950), $337-404$.

[2] Hida, T., Generalized multiple Wiener integrals, Proc. J. Acad., 54, Ser. A, (1978), 55-58.

[3] - Analysis of Brownian functionals, Carleton Math. Lec. Notes No.13, 2nd Ed. (1978) [c] Hida, T.: Brownian motion, Springer-Verlag, New York, Heidelberg, Berlin (1980).

[4] - Causal calculus of Brownian functionals and its applications, Statistics and related topics, ed. M. Csörgó et al. North Holland Publishing Company (1981).

[5] - White noise analysis and its applications, Proceedings of the International Mathematical Conference, Singapore 1981, (1982).

[ 6 ] Hida, T. and Ikeda, N., Analysis on Hilbert space with reproducing kernel arising from multiple Wiener integral, Proc. 5th Berkeley Symp. on Math. Stat. and Prob. Vol. II, Part 1, (1967), 117-143.

[ 7 ] Ito, K., Multiple Wiener integral, J. Math. Soc. Japan, 3 (1951), 157-169.

[ 8 ] Ito, Y., On a generalization of non-linear Poisson functionals, Math. Rep. Toyama 
Univ., 3 (1980), 111-122.

[ 9 ] - Generalized Poisson functionals, Prob. Th. Rel. Fields, 77 (1988), 1-28.

[10] Kubo, I. and Takenaka, S., Calculus on Gaussian white noises I, Proc. Japan Acad., 56 Ser. A, No. 8, (1980), 376-380.

[11] —- Calculus on Gaussian white noises II, Proc. Japan Acad., 56 Ser. A, No. 9, (1980), 411-416.

[12] - Calculus on Gaussian white noises III, Proc. Japan Acad., 57 Ser. A, No. 9 (1981), 433-437.

[13] — Calculus on Gaussian white noises IV, Proc. Japan Acad., 58 Ser. A, No. 9 (1982), 186-189.

[14] Kuo, H.-H., Gaussian measures in Banach spaces, Lect. Notes in Math., 463, Springer-Verlag, (1975).

[15] Segal, I., Tensor algebra over Hilbert spaces I, Trans. Amer. Math. Soc., 81 (1956), 106-134.

[16] - Tensor algebra over Hilbert spaces II, Ann. of Math., 63 (1956), 160-175.

[17] Wick, G. C., The evaluation of the collision of matrix, Phys. Rev., 80 (1950), 268-272.

[18] Wiener, N. and Wintner, A., Discrete chaos, Amer. J. Math., 65 (1943), 279-298.

Y. Ito

Nagoya University College of Medical Technology

Higashi-ku, Nagoya 461

Japan

I. Kubo

Hiroshima University

Naka-ku, Hiroshima 730

Japan 